\title{
Pre-metastatic niche drives breast cancer invasion by modulating MSC homing and CAF differentiation
}

\author{
Neha Saxena ${ }^{\mathrm{a}, \mathrm{b}}$, Garvit Bhardwaj ${ }^{\mathrm{c}}$, Sameer Jadhav ${ }^{\mathrm{a}}$, Hamim Zafar ${ }^{\mathrm{d}, \mathrm{e}}$, and Shamik Sen ${ }^{\mathrm{b}, \grave{\ddagger}}$ \\ ${ }^{a}$ Department of Chemical Engineering, IIT Bombay \\ ${ }^{\mathrm{b}}$ Department of Biosciences and Bioengineering, IIT Bombay \\ ${ }^{c}$ Department of Electrical Engineering, IIT Kanpur \\ ${ }^{\mathrm{d}}$ Department of Computer Science and Engineering, IIT Kanpur \\ eDepartment of Biological Sciences and Bioengineering, IIT Kanpur \\ ‡Corresponding Author: shamiks@iitb.ac.in
}

\begin{abstract}
The extent to which cancer-associated alterations in extracellular matrix stiffness influences the crosstalk between cancer cells and mesenchymal stem cells (MSCs) remains unclear. By analyzing multiple singlecell RNA sequencing datasets, we establish the existence of a cell sub-population co-expressing MSC and cancer associated fibroblast (CAF) markers in highly aggressive triple-negative breast cancers in primary tumor, secondary sites, and in circulatory tumor cell clusters. Using hydrogels of varying stiffness corresponding to different stages of cancer progression, we show that on pre-metastatic stroma mimetic $2 \mathrm{kPa}$ gels, MDA-MB-231 breast cancer cell secreted conditioned media drives efficient MSC chemotaxis and induces stable CAF differentiation in a TGF $\beta$ /contractility-dependent manner. In addition to enhancing cancer cell proliferation, $2 \mathrm{kPa}$ CAFs maximally boost local invasion and confer resistance to flow-induced shear stresses. Together, our results suggest that homing of MSCs at the pre-metastatic stage and their differentiation into CAFs actively drives breast cancer invasion and metastasis.
\end{abstract}

Key words: mesenchymal stem cells (MSCs); scRNAseq analysis; ECM stiffness; cancer associated fibroblast (CAF); differentiation; TGF $\beta$; cancer invasion; shear stress. 


\section{Introduction} available under aCC-BY-NC-ND 4.0 International license.

Of the multiple types of cells present in the tumor microenvironment (TME), an accumulating body of evidence has demonstrated the importance of cancer associated fibroblasts (CAFs) in actively driving cancer progression by fostering tumor growth and invasion via matrix metalloproteinase (MMP) mediated remodeling of the extracellular matrix (ECM) [1-4]. While increased secretion of fibrillar collagen, fibronectin and laminin lead to stromal stiffening and desmoplasia, force-mediated remodeling of synthesized ECM leads to formation of linearized tracks thereby promoting cancer invasion [5]. Though most CAFs express the myofibroblast marker $\alpha$ smooth muscle actin $(\alpha S M A)$, considerable phenotypic heterogeneity exists within CAFs indicative of existence of multiple CAF sub-types with variable expression of fibroblast activation protein $(F A P)$, fibroblast specific protein $(F S P)$ and platelet derived growth factor receptor $\beta(P D G F R \beta)[6,7]$. In breast cancer, in comparison to less aggressive luminal sub-types, more aggressive HER2+ and triple negative breast cancers (TNBCs) have been shown to harbour CAFs exhibiting elevated levels of FAP, $\alpha S M A$, $P D G F R \beta$ and $\beta 1$ integrins [8].

Though the origin of CAFs remains debatable, several studies have identified mesenchymal stem cells as a potential source of CAFs [9-11]. Mesenchymal stem cells (MSCs) represent one of the prominent cell-types that home at the site of tumors in response to factors released by cancer cells [12, 13]. MSC homing has been documented in multiple types of cancers including breast, lung and pancreatic cancers [14-16]. Both MSCs as well as adipocyte derived stem cells (ASCs) are known to differentiate into CAFs upon exposure to cancer cell secreted transforming growth factor $\beta$ (TGF $\beta$ ) [17]. MSCs, differentiated to CAFs, have been reported to increase the metastatic ability of weakly metastatic breast cancer cells with MSC secreted CCL5 implicated in inducing metastasis by enhancing cancer stem cell properties [9]. When co-cultured with breast cancer cells, MSCs have shown to exhibit higher homing through placental growth factor (PGF) and cancer metastasis through hypoxia inducible factor (HIF) [18]. In addition to secreting ECM proteins such as collagen and fibronectin [19], MSCs also play a crucial role in driving matrix stiffening through upregulation of lysyl oxidase (LOX) [18].

In epithelial cancers including breast, pancreatic and lung cancer, cancer aggressiveness and survival is associated with increased deposition, alignment and crosslinking of fibrillar collagens leading to several-fold increase in stromal stiffness [20, 21]. While MSCs are known to respond to matrix stiffness and differentiate into multiple lineages [22], the influence of stiffness in regulating MSC differentiation into CAFs is less understood. In a recent study, Keely and co-workers showed that upon exposure to cancer conditioned media (CM), MSCs differentiate into CAFs in a stiffness-dependent manner and drive cancer cell proliferation by secreting prosaposin [23]. However, the temporal dynamics of MSC homing at the site of tumors remains incompletely understood. Moreover, since stiffness regulates cancer cell secretome, it is likely that the response of MSCs to cancer CM is stiffness-dependent.

In this work, we address the importance of stiffness-dependent crosstalk between MSCs and MDA-MB-231 breast cancer cells in mediating MSC chemotaxis, MSC differentiation to CAFs and their subsequent in- 
volvement in cancer invasion. Based on analysis of single-cell RNAseq (scRNAseq) datasets from multiple breast cancer patients, we first show that aggressive triple negative breast cancers (TNBCs) harbour a subpopulation of cells exhibiting MSC/CAF markers. Using polyacrylamide gels of stiffnesses mimicking that of normal, pre-metastatic and metastatic stroma, we then show that MSC chemotaxis and CAF differentiation are optimal on pre-metastatic stroma-mimetic $2 \mathrm{kPa}$ gels in a TGF $\beta$-dependent, contractility-dependent manner, with CAF differentiation stable on $2 \mathrm{kPa}$ gels. Next, we illustrate the role of $2 \mathrm{kPa}$ CAFs in promoting cancer cell proliferation, stromal invasion and in sustaining physiological shear stresses. Finally, we perform analysis of scRNAseq data from circulators tumor cells (CTCs) and metastatic tumors to establish the presence of MSC/CAF expressing cells in CTC clusters and secondary tumors. In addition to illustrating the prominent role of MSCs in mediating cancer metastasis via stiffness-dependent homing at the primary tumor and differentiation into CAFs, our results implicates the pre-metastatic niche as an active driver of cancer progression.

\section{Results}

\section{Presence of a MSC/CAF signature in triple negative breast cancers (TNBCs)}

Among the four molecular subtypes of breast cancer, the TNBC sub-type is the most aggressive subtype in terms of clinical course and is known to be characterized by extensive inter-tumor as well as intra-tumor heterogeneity [24]. The aggressiveness of TNBC is characterized by early onset of disease, higher incidence of metastasis, greater relapse rate and shorter overall survival [25]. In order to evaluate if the stromal component of TNBCs harbour MSCs that contribute to higher invasiveness observed in TNBC, we analyzed scRNAseq data from 551 cells that were sampled from 11 breast cancer patients [26]. These cells were divided into a group consisting of 214 cells from 5 TNBC patients and another group consisting of 337 cells that were sampled from patients with other molecular subtypes (2 luminal A, 1 luminal B and 3 HER2-positive patients). Within each group of cells, we identified transcriptionally distinct cell populations by performing clustering using Seurat [27] and subsequently tested the clusters for enrichment of known MSC markers including CD90, $C D 106$ [28] and CD140b [29]. While 3 clusters were identified in the group consisting of TNBC cells (Fig. 1A), a subset of 10 cells in cluster 1 (green) expressed the MSC markers (Fig. 1C). In contrast, cells from group 2 were divided into 7 cell types (Fig. 1B), none of which expressed the MSC markers (Fig. 1D).

For the TNBC group, we selected the 10 cells expressing MSC markers and performed differential gene expression analysis by comparing with all other TNBC cells (Supp. Fig. 1A). This analysis revealed CD90 ( THY1) to be one of the top differentially expressed genes in these cells (Supp. Fig. 1B). In addition, other MSC markers including COL1A1 [30], COL1A2 [31], POSTN [32] were found to be differentially expressed in these 10 cells indicating these cells to be MSCs that invaded the tumor stroma. The putative MSCs identified from the scRNAseq dataset were further tested for the expression of CAF markers. The putative 
bioRxiv preprint doi: https://doi.org/10.1101/2021.01.12.426460; this version posted January $13,2021$. The copyright holder for this preprint

(which was not certified by peer review) is the author/funder, who has granted bioRxiv a license to display the preprint in perpetuity. It is made available under aCC-BY-NC-ND 4.0 International license.

MSCs were found to express known CAF markers including ACTA2 (also known as $\alpha S M A$ ), FAP, CAV1, CD29, TAGLN, COL1A2, DCN in both the data sets (Fig. 1E, Supp. Fig. 1C) and these genes were further enriched in differential expression analysis (Supp. Figs. 1B). Along with the expression of CAF associated genes, the putative MSCs also express markers associated with invasion (MMP2), ECM stiffening (LOX) as well as markers associated with bone metastasis (CTGF, IGF1) (Supp. Fig. 1C).

We further checked another scRNAseq TNBC dataset for the presence of MSCs [33]. This dataset consisted of 24271 cells that were sampled from five primary TNBC patients. Clustering analysis with Seurat identified 20 cell type clusters of which three clusters (Clusters 6, 11 and 16) expressed different MSC markers (CD90, CD106, PDGFRB) (Supp. Fig. 2A). As before, MSC, CAF and invasion markers were found to be differentially expressed in the above three clusters (Supp. Figs. 2B, 3). To further characterize the lineage relationships between the cells expressing MSC/CAF signature in TNBC, 3 clusters were used from this dataset for trajectory inference. Differentiation trajectory of the cells in these 3 clusters was reconstructed using Monocle 3 [34], which inferred a branched trajectory consisting of three major branches (Supp. Fig. 4A). The cells were pseudotemporally ordered by selecting the cells expressing MSC markers CD105 and Stro-1 as the initial population in the trajectory. Cells in Branch 1 were the closest to the MSCs expressing the MSC marker CD90 as well as the CAF markers ACTA2, CAV1 and S100A4 (also referred to as FSP1) (Supp. Fig. 4B). These cells further differentiated into two branches, with Branch 2 expressing both MSC (COL1A1, CD90) and CAF markers (ACTA2, DCN, COL1A2) and Branch 3 expressing CAF markers (FSP1, COL1A2, $C A V 1)$. Collectively, the trajectory analysis suggests that the sub-population of cells expressing MSC markers in TNBC tissue differentiate into CAFs as well as cells that co-express CAF and MSC markers.

\section{Stiffness-modulated cancer conditioned media (CCM) regulates MSC chemo- taxis}

Based on the above anyalsis, we hypothesize that cells expressing MSC/CAF markers correspond to MSCs which home at the site of primary tumor and undergo partial differentiation into CAFs. Matrix stiffening is a hallmark of breast cancer progression [35-37]. To determine how biophysical alterations in ECM mechanics associated with cancer progression is temporally correlated with homing of MSCs at the site of primary tumor, we probed the influence of stiffness-dependent secretome of cancer cells in mediating MSC chemotaxis. Specifically, MSC chemotaxis was studied in response to factors secreted by MDA-MB-231 breast cancer cells cultured on polyacrylamide gels mimicking bulk stiffness of normal mammary stroma ( $0.5 \mathrm{kPa})$, pre-metastatic tumor stroma $(\approx 2 \mathrm{kPa})$, and metastatic tumor stroma $(\approx 5 \mathrm{kPa})[35,36]$. Chemotaxis experiments were performed using a microfluidic device comprising of two large side channels connected by several transverse channels wherein introduction of a chemokine in one of the side channels leads to establishment of a stable chemical gradient, leading to MSC chemotaxis (Fig. 2A) [38]. While MSCs mixed with collagen gels were seeded on one of the side channels in plain MSC culture media, the remaining portion was filled with only collagen gel (cell free side). 
Upon adhesion of MSCs to the surrounding collagen matrix, cancer conditioned media (CCM) collected from MDA-MB-231 cells cultured on gels (i.e., $0.5 \mathrm{kPa} \mathrm{CM}, 2 \mathrm{kPa} \mathrm{CM}$ and $5 \mathrm{kPa} \mathrm{CM}$ ) and from tissue culture plates (i.e., TCPCM) was introduced at the cell free side. Experiments were performed by varying the concentration of collagen gels so as to generate matrices of varying pore sizes (Fig. 2B). Quantification of trajectories of individual MSCs (red lines) along the transverse channels (white dotted lines) tracked by labeling nuclei (blue) revealed marked differences in MSC motility across the different conditions (Fig. 2C). While cell motility was collectively influenced by collagen concentration and CCM composition, across all the collagen gels, fastest migration was observed in the presence of $2 \mathrm{kPa}$ CM (Fig. 2D). Highest cell persistence was observed in the presence of $2 \mathrm{kPa} \mathrm{CM}$ and $5 \mathrm{kPa} \mathrm{CM}$. Together, these results suggest that $2 \mathrm{kPa} \mathrm{CM}$ optimally induces MSC chemotaxis.

\section{Stiffness regulates MSC differentiation into CAFs in a TGF $\beta$-dependent manner}

To next probe how stiffness of the tumor microenvironment influences the fate of MSCs which home at the site of primary tumors, MSCs were cultured for 7 days on $0.5 \mathrm{kPa}, 2 \mathrm{kPa}$ and $5 \mathrm{kPa}$ gels using conditioned media (CM) composed of normal MSC media and CCM in 1:1 ratio (Fig. 3A). MSCs cultured on the respective gels in regular MSC media served as controls. While initial spreading at Day 1 was stiffness-dependent with CM making no difference, CM-incubated MSCs exhibited increased spreading on 2 and $5 \mathrm{kPa}$ gels after 7 days in culture (Fig. 3B, C). In comparison, MSC motility was found to be sensitive to presence of CM on $2 \mathrm{kPa}$ gels on Day 1 itself, with further increase in motility detected on 2 and $5 \mathrm{kPa}$ gels at Day 7 (Fig. 3C). After 7 days in culture, in comparison to $0.5 \mathrm{kPa}$ gels, cell spreading and motility were higher on 2 and $5 \mathrm{kPa}$ gels in the presence of CM. Additionally, tracking of cell proliferation over a period of 7 days revealed highest proliferation on $2 \mathrm{kPa}$ substrates (Supp. Figs. 5A, B).

Higher sensitivity of MSC spreading and motility on $2 \mathrm{kPa}$ gels in the presence of CM correlated with increased cytoskeletal organization and higher cortical stiffness in these cells (Supp. Figs. 5C, D). Since expression of the MSC marker Stro-1 was downregulated after 1 week in the presence of CM on both 2 and $5 \mathrm{kPa}$ gels (Supp. Figs. 6Ei, Eii), we hypothesized that the observed phenotypic changes were indicative of MSC differentiation

into CAFs. Consistent with this, expression of CAF markers $\alpha$-SMA and FAP $[6,7,39]$ were elevated on 2 $\mathrm{kPa}$ gels in the presence of $\mathrm{CM}$, but not on $5 \mathrm{kPa}$ gels (Fig. 3D). Further, expression of the nuclear marker Lamin A ( LMNA) associated with MSC differentiation increased 5-fold on $2 \mathrm{kPa},+\mathrm{CM}$ condition (Fig. 3D). Consistent with these observations, immunostaining for $\alpha-S M A$ and $L M N A$ revealed highest expression on 2 $\mathrm{kPa},+\mathrm{CM}$ condition (Figs. 3E, F).

Since TGF $\beta$ is known to induce CAF differentiation [17], we first checked levels of TGF $\beta$ in CCM collected from the different gels, with cell culture media used as blanks. Maximum upregulation of CAF markers on $2 \mathrm{kPa}$ gels was associated with highest TGF $\beta$ level on $2 \mathrm{kPa}$ gels (Fig. 3G). To establish the importance of $\mathrm{TGF} \beta$ in driving CAF differentiation, experiments were performed wherein MSCs were incubated with CM 
in the presence of TGF $\beta$ inhibitor (Supp. Fig. 6F). Antibody-mediated blocking of TGF $\beta$ receptor led to $\geq 50 \%$ drop in $\alpha$-SMA levels on both 2 and $5 \mathrm{kPa}$ gels and $\approx 30 \%$ drop in $L M N A$ levels on $2 \mathrm{kPa}$ gels (Fig. $3 \mathrm{H}$, Supp. Figs. 6Gi, Gii). Collectively, these results suggest that MSC differentiation to CAFs is driven by TGF $\beta$ present in the CCM, with highest TGF $\beta$ secreted by MDA-MB-231 cells on $2 \mathrm{kPa}$ gels.

\section{CAF differentiation is contractility-dependent and is stable on $2 \mathrm{kPa}$ gels}

In line with highest $\alpha$-SMA expression on $2 \mathrm{kPa}$ gels, $2 \mathrm{kPa}$ CAFs possessed highest pMLC levels in the presence of CM (Figs. 4A, B) and exerted highest tractions (Figs. 4C, D, Supp. Fig. 6H). Since stiffnessdependent differentiation requires non-muscle myosin II-dependent stiffness-sensing [22], to probe the role of contractility in mediating MSC differentiation into CAFs, experiments were performed wherein MSCs were cultured in $\pm \mathrm{CM}$ in the presence of the non-muscle myosin II inhibitor blebblistatin (Bleb) for a period of 7 days (Fig. 4E). While Bleb treatment did not alter $\alpha-S M A / L M N A$ expression in the absence of CM, in cells cultured in the presence of CM, in line with significant reduction in pMLC levels in Bleb-treated cells (Supp. Figs. 6Ii, Iii), a dramatic drop in both $\alpha-S M A$ and $L M N A$ intensities were observed (Figs. 4E, F). Together, these results highlight the role of contractility in mediating CM induced differentiation of MSCs into CAFs.

To next assess the stability of MSC differentiation into CAFs, MSC-differentiated CAFs were cultured for another 5 days in culture in low serum media in the absence of CM (Fig. 4G). While the drop in $\alpha-S M A / L M N A$ levels on $2 \mathrm{kPa}$ gels were marginal and statistically insignificant, $\alpha-S M A$ levels dropped significantly on $5 \mathrm{kPa}$ gels (Fig. 4H). To further probe the sensitivity of CAF marker expression to contractile inhibition, CAFs were cultured for 3 days in the presence of Bleb in the absence of CM (Supp. Fig. 7A). Remarkably, levels of pMLC and $\alpha-S M A$ remained relatively unchanged on $2 \mathrm{kPa}$ gels, but dropped significantly on $5 \mathrm{kPa}$ gels (Supp. Figs. 7Bi, Bii). Collectively, these results suggest that $2 \mathrm{kPa}$ substrates induce stable differentiation of MSCs into CAFs in a contractility-dependent manner.

\section{$2 \mathrm{kPa}$ CAFs maximally enhance proliferation and invasiveness of cancer cells}

To next probe the role of CAFs in modulating cancer cell behavior, MDA-MB-231 cells were cultured on 2 and $5 \mathrm{kPa}$ gels in the presence of CAF secreted media, i.e., CAF conditioned media (CAFCM) (Fig. 5A). While CAFCM was found to induce increased cell spreading on both the gels (Figs. 5B, C), tracking of cell proliferation over a 7 day time period revealed fastest proliferation on $2 \mathrm{kPa}$ gels supplemented with CAFCM (Figs. 5D, E).

To next investigate the role of CAFs in regulating cancer invasion, spheroids $(S)$ were prepared using hanging drop method with MDA-MB-231 cells alone $\left(S_{\mathrm{M}}\right)$ or by combining MDA-MB-231 cells with $2 \mathrm{kPa}$ CAFs or $5 \mathrm{kPa}$ CAFs ( $S_{\mathrm{MF} 2}$ and $S_{\mathrm{MF} 5}$, respectively) (Fig. $5 \mathrm{~F}$ ). The sizes of the three different types of spheroids were similar with most cells within the spheroids being viable (Supp. Figs. 7C-E). Invasiveness of spheroids was 
assessed by implanting single spheroids in 3D collagen gels and tracking the extent of outward cell scattering over a period of 7 days (Fig. 5G). Quantification of the area invaded by cells migrating out from the spheroids revealed maximum invasiveness of spheroids formed from $2 \mathrm{kPa}$ CAFs, i.e., $S_{\mathrm{MF} 2}$ spheroids (Fig. $\left.5 \mathrm{H}\right)$.

Recent literature has demonstrated the role of CAFs in driving collective invasion of cancer cells [40]. To probe the role of $2 \mathrm{kPa}$ and $5 \mathrm{kPa}$ CAFs in leading cancer invasion, CAFs were stained with CellTracker Red and MDA-MB-231 with CellTracker Green to visualize the spatial position of the two cell types within the spheroids and also during the outward invasion. Within the heterospheroids implanted in collagen gels (i.e., Day 0 spheroids), the extent of CAF enrichment at the outer periphery was clearly observed in $S_{\mathrm{MF} 2}$ spheroids, but not in $S_{\mathrm{MF} 5}$ spheroids (Supp. Fig. $7 \mathrm{~F}$ ). Consistent with this, while the invasive front of $S_{\mathrm{MF} 2}$ spheroids comprised primarily of $2 \mathrm{kPa}$ CAFs, the invasive front of $S_{\mathrm{MF} 2}$ spheroids were populated by both $5 \mathrm{kPA}$ CAFs as well as MDA-MB-231 cells (Fig. 5I). Highest invasiveness of $S_{\mathrm{MF} 2}$ spheroids was associated with highest expression of $M M P 2$ in $2 \mathrm{kPa}$ CAFs, with $M M P 9$ expression remaining unaltered (Fig. 5J). In line with highest $M M P 2$ expression, quantification of the extent of collagen degradation visualized using DQ collagen revealed maximum degradation (i.e., fluorescence) by $2 \mathrm{kPa}$ CAFs (Figs. 5K-M). Taken together, these results suggest that in addition to promoting cancer cell proliferation, $2 \mathrm{kPa}$ CAFs drive cancer invasion via increased matrix degradation.

\section{Spheroids formed using $2 \mathrm{kPa}$ CAFs are more adhesive and resistant to shear stresses}

Thus far, our results suggest that $2 \mathrm{kPa}$ CAFs enhance stromal invasiveness of cancer cells. Cancer cell metastasis involves entry into the vasculature, adhesion to the endothelium and subsequent extravasation. To probe the possible role of CAFs in modulating adhesion and shear resistance of heterospheroids, integrin profiling of CAFs and adhesivity of heterospheroids were performed. Integrin profiling of CAFs revealed significant upregulation in expression of $\beta 1$ and $\beta 3$ integrins in $2 \mathrm{kPa}$ CAFs, but not in $5 \mathrm{kPa}$ CAFs (Figs. 6A, Bi, Bii). To next probe the consequence of increased integrin expression in $2 \mathrm{kPa}$ CAFs in modulating adhesion of cancer cells, adhesion rate and adhesion strength of $S_{\mathrm{M}}, S_{\mathrm{MF} 2}$ and $S_{\mathrm{MF} 5}$ spheroids were assessed. For adhesion experiments, spheroids allowed to attach on to fibronectin-coated coverslips were washed after 1 hr to check the proportion of spheroids that remained attached (Fig. 6C). While the proportion of attached spheroids was comparable between $S_{\mathrm{M}}$ and $S_{\mathrm{MF} 5}$ spheroids, the number was higher for $S_{\mathrm{MF} 2}$ spheroids (Figs. 6Di, Dii). To assess the strength of adhesion, spheroids were allowed to attach onto fibronectin-coated coverslips for 2 hrs, and then incubated with warm trypsin to assess the time required for detachment $\left(\tau_{\text {detach }}\right)$ (Fig. 6E), with the exact time of detachment determined based on movement of spheroid centroids from their initial positions (Fig. 6Fi). The detachment duration, $\tau_{\text {detach }}$, given by the expression $\tau_{\text {detach }}=\left(\tau^{*}-120\right)$ min, serves as a surrogate for adhesion strength with higher values indicative of stronger adhesion. Quantification of detachment timescales revealed that $S_{\mathrm{MF} 2}$ spheroids exhibited stronger adhesion and took nearly twice as long to detach in comparison to $S_{\mathrm{M}}$ and $S_{\mathrm{MF} 5}$ spheroids (Fig. 6Fii). 
To further test if $S_{\mathrm{MF} 2}$ spheroids-which exhibit faster attachment and stronger adhesion-also exhibit greater resistance to shear, shear flow experiments were performed using a straight microfluidic channel with an inlet port, an outlet port, and an intermediate port for introducing spheroids (which was sealed after introducing spheroids through it) (Fig. 6G). After allowing the spheroids to attach onto the fibronectin-coated base of the microfluidic channel for $8 \mathrm{hrs}$, the inlet port was connected to a syringe pump. For a physiologically relevant flow rate of $400 \mu \mathrm{l} / \mathrm{min}$ [41], simulations predicted buildup of shear stresses upto $20 \mathrm{~Pa}$ (Fig. $6 \mathrm{H})$. Plotting of shear stresses along the dotted white lines suggest spheroids situated at the middle of the long channel $(x=400 \mu \mathrm{m})$ are subjected to shear stresses of $\approx 20$ Dynes $/ \mathrm{cm}^{2}$. Consistent with longer trypsin de-adhesion timescales, the time taken by spheroids to detach under shear, i.e., $\tau_{\text {shear }}$-indicative of strength of adhesion-was nearly twice as higher for $S_{\mathrm{MF} 2}$ spheroids compared to that of $S_{\mathrm{M}}$ and $S_{\mathrm{MF5}}$ spheroids (Figs. 6I, J). Collectively, these results suggest that increased integrin expression on $2 \mathrm{kPa}$ CAFs makes $S_{\mathrm{MF} 2}$ spheroids more adhesive and resistant to shear stresses.

\section{Presence of MSCs/CAFs in circulating tumor cell (CTC) clusters and in sec- ondary metastases}

Cancer metastasis is mediated by clusters of circulating tumor cells (CTCs) with CTC clusters possess 50fold higher chance of initiating metastasis compared to individual CTCs. To evaluate if MSCs and/or CAFs are present in CTC clusters, we analyzed scRNAseq data from CTCs isolated from invasive breast cancer patients and MMTV-PyMT mouse models [42]. The dataset consisted of 61 CTCs which were preprocessed and clustered into distinct cell types using Seurat. We identified three CTC clusters each of which expressed MSC markers CD29, CD73, CD166 (all clusters), CD105 (clusters 0 and 1), VCAM1 (cluster 0) and CD45 (cluster 1). The clusters also showed enrichment of CAF markers including ACTA2, FSP1, CAV1 and TAGLN. Apart from these, the CTCs also expressed CD44, LMNA, MMP9 as well as the bone metastasis marker CTGF (Fig. 7A and Supp. Fig. 8A). This suggests the presence of cell types other than cancer cells in CTC clusters which might help them to survive in blood flow and effectively metastasize to secondary sites.

CD44-mediated homophilic interactions have been shown to drive multicellular aggregation in TNBCs [43]. Given the prominent expression of $C D 44$ in all the three CTC clusters, and the presence of cells exhibiting MSC/CAF marker expression, we next checked CD44 expression in $2 \mathrm{kPa}$ and $5 \mathrm{kPa}$ CAFs. In comparison to baseline CD44 expression in MSCs, CD44 expression increased significantly in both 2 and $5 \mathrm{kPa}$ CAFs with highest expression in $2 \mathrm{kPa}$ CAFs (Fig. 7B). Though CD44 expression in cancer cells was found to be 10-15 fold higher compared to CAFs (Supp. Figs. 7Gi, Gii), staining of $S_{\mathrm{MF} 2}$ spheroids invading collagen gels revealed comparable levels of $C D 44$ at the interface of CAFs and MDA-MB-231 cells in contact at the invasive front (Supp. Fig. $7 \mathrm{H}$ ).

To finally test if CTCs expressing MSC/CAF signature were associated with metastasis, we integrated single- 
bioRxiv preprint doi: https://doi.org/10.1101/2021.01.12.426460; this version posted January $13,2021$. The copyright holder for this preprint

(which was not certified by peer review) is the author/funder, who has granted bioRxiv a license to display the preprint in perpetuity. It is made available under aCC-BY-NC-ND 4.0 International license.

cell RNA sequencing data from primary and metastatic tumor cells (375 cells in total) captured during seeding of micrometastasis in patient-derived-xenograft models of breast cancer [44] with the $61 \mathrm{CTC}$ scRNAseq data [42]. To eliminate batch effects, we performed anchor-based integration using Seurat [45] and clustered the cells. The cells were clustered into two groups out of which the larger cluster (cluster 0) consisted of 54 CTCs, 121 primary and 129 metastatic tumor cells (Supp. Fig. 8B), whereas the other cluster only contained 7 CTCs and was dominated by 71 primary and 54 metastatic cells. The cluster harbouring both CTCs and metastatic cells in larger proportion (i.e., cluster 0) showed enrichment for MSC markers (CD73), CAF markers (ACTA2, CAV1) and other markers (CD44 and LMNA). This analysis suggests that CTCs that express MSC/CAF signature were also associated with primary and metastatic cells expressing the same markers.

\section{Discussion}

In this study, by analyzing publicly available scRNAseq data from breast cancer patients, we first correlated the aggressiveness of TNBCs with presence of a sub-population of cells exhibiting MSC/CAF signature. We then showed that stiffness-modulated CCM regulates MSC chemotaxis and differentiation into CAFs. Next, we demonstrated the enhanced capacity of $2 \mathrm{kPa}$ CAFs in enhancing invasiveness of cancer cell and imparting resistance to flow-induced shear stresses. Finally, by analyzing scRNAseq data from CTCs, we showed that CTC clusters harbour cells exhibiting MSC/CAF markers. Based on our findings, we propose a model wherein cancer progression is associated with MSC homing to tumors at the pre-metastatic stage, differentiation into CAFs, and subsequent CAF driven invasion and metastasis (Fig. 7C). Our results suggest that the combination of physical and chemical cues present in the pre-metastatic niche actively drives cancer progression by efficiently recruiting MSCs and stably differentiating them into CAFs. In addition to driving local invasion, our results suggest that MSC differentiated CAFs may aid in secondary organ colonization through increased adhesion under shear.

The tumor microenvironment (TME) represents a dynamic environment undergoing continuous alterations that collectively drive cancer aggressiveness. In addition to dynamic alterations in ECM properties, the presence of of multiple cell types including immune cells, resident fibroblasts, pericytes, CAFs as well as MSCs highlight the cell level heterogeneity within the TME $[8,46]$. By analyzing publicly available scRNAseq datasets, we have established the presence of cells co-expressing MSC/CAF makers in TNBCs, but not in other subtypes of breast cancer. Trajectory analysis suggests that MSC differentiation can lead to generation of CAFs as well as an intermediate differentiated phenotype where both MSC and CAF markers are co-expressed. However, the physical cues determining these differentiation trajectories remain unclear. By probing stiffness-mediated bi-directional crosstalk between MSCs and cancer cells, we establish the importance of the pre-metastatic niche in giving rise to this mixed MSC/CAF signature. In our experiments, conditioned media secreted by cancer cells on pre-metastatic stroma-mimetic $2 \mathrm{kPa}$ gels optimally induced MSC chemotaxis. MSCs are known to migrate in response to several tumor site-specific soluble factors 
bioRxiv preprint doi: https://doi.org/10.1101/2021.01.12.426460; this version posted January $13,2021$. The copyright holder for this preprint (which was not certified by peer review) is the author/funder, who has granted bioRxiv a license to display the preprint in perpetuity. It is made available under aCC-BY-NC-ND 4.0 International license.

including interleukin-6 (IL-6), IL-1, TGF $\beta 1$, epidermal growth factor (EGF) and stromal derived factor 1 (SDF-1) $[12,47]$. Fastest MSC chemotaxis induced by $2 \mathrm{kPa}$ cancer conditioned media (CCM) may be partly attributed to elevated TGF $\beta$ levels. In a recent study on stiffness-dependent modulation of secretome of breast cancer cells, sphingosine-1-phosphate (S1P) was identified as an important bioactive lipid secreted by MDA-MB-231 cells to a greater extent on softer substrates [48]. Interestingly, S1P is a potent chemoattractant for MSCs driving their exit from the bone marrow to the peripheral blood [49]. Thus, increased MSC chemotaxis in response to $2 \mathrm{kPa} \mathrm{CCM}$ may be driven by the presence of multiple factors in the CCM and warrant a detailed proteomic analysis.

CAFs represent one of the most abundant cell types in the TME and constitute a heterogeneous population of mesenchymal-origin cells exhibiting both tumor-promoting and tumor-suppressive effects. Microenvironmental heterogeneity between low grade and high grade tumors has been shown to induce formation of different subtype of CAFs [50]. In breast cancer, four different CAF subsets have been identified based on expression of integrin $\beta 1, \alpha-S M A, F S P 1, F A P, P D G F R$ and $C A V 1$ [8]. In a recent study, Keely and co-workers demonstrated that CCM induces stiffness-dependent MSC differentiation into CAFs expressing high $\alpha-S M A$ [23]. However, the CCM used in this study was collected from cancer cells cultured on tissue culture plastic (TCP). Here, we show that stiffness-dependent CCM directly influences the expression profile of differentiated CAFs with a $\alpha$-SMA ${ }^{h i} \mathrm{FAP}^{h i}$ signature observed on $2 \mathrm{kPa}$ gels. Our findings are consistent with a recent study wherein ECM composition and stiffness were identified as factors regulating TGF $\beta$-induced differentiation of murine pulmonary fibroblasts into CAFs with $\mathrm{FAP}^{h i}$ expressing CAFs observed on soft substrates [51]. FAP is a postprolyl peptidase capable of degrading both collagen and gelatin, and is involved in matrix turnover [52]. High FAP expression has been correlated with distant metastasis and poor prognosis in several solid tumors including breast cancer [37, 53-55]. MSC differentiation into $\mathrm{FAP}^{h i} \mathrm{CAFs}$ on $2 \mathrm{kPa}$ gels thus highlights the role of the pre-metastatic niche in actively driving cancer progression.

Cancer cell secreted TGF $\beta$ leads to the conversion of stromal fibroblasts into myofibroblastic CAF subtype by inducing expression of $\alpha-S M A$ and FAP [3]. Consequently, blocking TGF $\beta$ in-vivo inhibits the formation of this myofibroblastic CAF subtype [56]. Highest $\alpha-S M A$ and $L M N A$ expression on $2 \mathrm{kPa}$ gels coincided with highest levels of TGF $\beta$ secreted on these gels. In line with this drop in $\alpha-S M A$ levels upon TGF $\beta$ receptor blocking highlights the role of stiffness-dependent TGF $\beta$ signaling in mediating CAF differentiation. TGF $\beta$ is known to activate Rho/ROCK signaling pathway and induces expression of actin associated proteins such as calgizzarin, cofilin, and profillin [57]. Increased expression of $\alpha-S M A$ participates in the formation of stress fibres, and contributes to increased cytoskeletal tension and contractile properties of CAFs [57, 58]. In line with this, $2 \mathrm{kPa}$ CAFs were found to possess highest levels of activated myosin (pMLC) and were the most contractile. Bleb-mediated inhibition of actomyosin contractility illustrates the importance of stiffness-sensing for MSC differentiation into CAFs. In addition, Bleb treatment has also been shown to inhibit conversion of latent $\mathrm{TGF} \beta$ to its active form which is required for CAF differentiation [59, 60]. CAFs have been shown to exhibit plasticity, i.e., they exhibit inter-conversion from one state to another [61, 62], may depend on the extent of differentiation. Highest $L M N A$ expression on $2 \mathrm{kPa}$ gels combined with relative insensitivity of 2 
$\mathrm{kPa}$ CAFs to Bleb treatment is indicative of stable CAF differentiation on $2 \mathrm{kPa}$ gels.

In comparison to $5 \mathrm{kPa}$ CAFs, $2 \mathrm{kPa}$ CAFs led to increased cancer cell proliferation and invasion. Increased proliferation of breast cancer cells may be driven by several CAF secreted factors including SDF-1, FGF1 and uPA [63]. Recently, Keely and co-workers showed that prosaponin secreted by MSCs on stiff substrates promotes proliferation of $4 \mathrm{~T} 1$ mouse mammary cancer cells via AKT activation [23]. In contrast to this study, higher cancer cell proliferation observed on $2 \mathrm{kPa}$ gels compared to $5 \mathrm{kPa}$ gels may be attributed to the use of CAFCM instead of MSCCM. Maximal invasiveness of $2 \mathrm{kPa}$ CAF-containing heterospheroids reported in our study may be attributed to the combination of higher $M M P / \alpha-S M A$ expression and increased contractility of $2 \mathrm{kPa}$ CAFs leading to a combination proteolytic and force-driven remodeling of the ECM. In vivo, $\alpha \mathrm{SMA}^{h i} \mathrm{FAP}^{h i} \mathrm{CAFs}$ drive cancer progression by LOX-mediated matrix stiffening and alignment generating matrices amenable for cancer invasion [64, 65]. In addition to path generation, CAFs have also been reported to physically pull out cancer cells from tumors through formation of heterotypic adhesions [40]. CD44+ fibroblasts have been shown to promote survival and drug resistance of cancer cells [66]. Given the ability of $C D 44$ to form homophilic adhesions [43], it is possible that CD44 also mediates adhesion between CAFs and MDA-MB-231 cells.

Secondary metastasis is mediated by CTC clusters which circulate in the blood stream, adhere to the endothelium and subsequently extravasate to seed secondary tumors, with CTC clusters possessing increased metastatic potential compared to single CTCs [67]. In addition to mediating stromal invasion, recent studies have documented the presence of CAFs in CTC clusters [68, 69]. Our analysis of scRNAseq data also suggests the presence of CAFs in CTC clusters in breast cancer patients. To overcome the shear stress from blood flow, cancer cells are known to associate with platelets [70]. In a recent study, King and co-workers demonstrated that CAFs confer fluid shear stress resistance to prostate cancer cells via intercellular contacts thereby maintaining viability and proliferative capacity of cancer cells [71]. Our results of increased adhesion and shear resistance of $S_{M F 2}$ spheroids are consistent with this study. The prominent expression of $C D 44$ observed in CTC clusters may not only contribute to maintaining cohesion of CTC clusters, but also mediate endothelial adhesion [72]. In addition to $C D 44$, endothelial adhesion may also be mediated by $\beta 1 / \beta 3$ integrins via binding to the fibronectin on the surface of endothelial cells $[73,74]$.

Different tumor types exhibit different secondary organ preference during metastasis. Consequently, identifying factors driving organ-specific metastasis represents an area of active research. Primary breast tumors are capable of metastasizing to several organs including lungs, liver, brain and bone, with bone frequently being the preferred site [75]. The presence of fenestrated endothelial cells and a discontinuous basal lamina in the bone marrow make it relatively easier for CTC clusters to seed bone metastasis [76]. In a seminal study, Massagúe and co-workers also demonstrated that MSC enriched triple negative breast tumors exhibit bone metastasis with MSC differentiated CAFs secreting high levels of CXCL12 and IGF1 [77]. Consistent with this, CTGF [78] and IGF1 [79], associated with bone metastasis, were also observed in the cells expressing MSC/CAF markers within TNBC tumors. These factors which are present at high levels in the bone marrow, 
in turn lead to establishment of clones with high Src activation and Src dependent PI3-Akt activation which increases the preference of breast cancer cells for the bone environment [77]. In addition, bone colonization may be mediated by $\beta 3$ integrins expressed both by CAFs and MDA-MB-231 cells, that can bind to osteopontin and vitronectin expressed by osteoblasts [80].

In conclusion, our study illustrates how stiffness-dependent crosstalk between MSCs and breast cancer cells drives cancer invasion through increased MSC homing, CAF differentiation and subsequent CAF-mediated invasion. Future studies targeting the crosstalk between MSCs and cancer cells at the pre-metastatic stage may lead to identification of therapeutic strategies for delaying disease progression.

\section{Materials \& Methods}

\section{scRNAseq data processing, clustering and visualization}

We first preprocessed the scRNAseq datasets by filtering out cells that have unique feature counts over 2500 or less than 200. The cells containing $>5 \%$ mitochondrial counts were further removed. The cells remaining after the pre-processing steps were subject to normalization, and scaling using the Seurat V3 package [27]. For clustering the cells, we first selected the top variable genes (2000) using the FindVariableFeatures function in Seurat. Principal component analysis (PCA) was performed for reducing the dimensions of gene-expression data to the top 20 principal components. For visualizing the cells, the PCA space representation of the cells were projected to 2D using Uniform Manifold Approximation and Projection (UMAP) algorithm. The FindNeighbors and FindClusters in Seurat V3 were used for clustering the cells on PCA space using the Shared Nearest Neighbor (SNN) algorithm. The resulting cell clusters were visualized on UMAP space using the DimPlot function in Seurat. The expression of marker genes on the clusters were visualized using FeaturePlot function. The differentially expressed genes in each cluster were inferred using the FindAllMarkers function in Seurat. DoHeatMap function was used for generating the expression heatmap for cell clusters and top 20 differentially expressed genes for all clusters.

\section{Trajectory inference and pseudotemporal ordering}

The differentiation trajectory of the cells expressing MSC/CAF markers was reconstructed using Monocle 3 [34]. In order to reconstruct the trajectory, first the selected cells were projected to a lower dimension using the reduceDimension() function in Monocle 3 which uses UMAP underneath. Next, the cells were partitioned using cluster_cells() function to look for potentially discontinuous trajectories. In our case, all the cells were grouped into 1 cluster. The learn_graph() function was used for learning a principal graph that delineates the possible trajectory of the cells. The pseudotime for the cells were calculated using the order_cells() function by selecting the cells expressing MSC markers (CD105 and Stro-1) as the initial population. The expression of the marker genes on the trajectory was visualized using the plot_cells() function. 


\section{Integration of scRNAseq datasets} available under aCC-BY-NC-ND 4.0 International license.

For integrating the CTC and metastatic cancer datasets, we first preprocessed and normalized the datasets using Seurat V3 package [45]. For each dataset, we selected the top 2000 highly variable genes using the FindVariableFeatures function in Seurat V3 with the 'vst' selection method. For batch effect elimination, anchors were inferred using the FindIntegrationAnchors function and based on the inferred anchors, the datasets were integrated using IntegrateData function. The integrated data was scaled, and dimension reduction, clustering and visualization steps were followed as described before.

\section{Cell culture \& Reagents}

Primary human mesenchymal stem cells (MSCs) were obtained from Lonza (Cat \# PT-2501) and MDAMB-231 human breast cancer cells from NCCS, Pune. MSCs were maintained in complete media consisting of low glucose Dulbecco's modified eagles medium (DMEM) (Himedia, Cat \# AL006A) supplemented with 16\% foetal bovine serum (FBS, Thermo Scientific, Cat \# 12662029), 1\% penicillin-streptomycin (Pen-strep, Sigma, Cat \# ) and 1\% L-glutamine (Thermo Scientific, Cat \# 35050061). MDA-MB-231 cells were cultured in DMEM-High Glucose (Himedia, Cat \# AL006) with 10\% FBS (Himedia, Cat \# 1112), 1\% pen-strep and 1\% L-glutamine. For TGF $\beta$ inhibition experiments, TGF $\beta$ neutralizing antibody (R\&D systems, DY240) was added at a concentration of $1.25 \mu \mathrm{g} / \mathrm{ml}$. Contractility inhibition experiments were carried out by adding the non-muscle myosin II inhibitor blebbistatin $(5 \mu \mathrm{M})$.

\section{Fabrication and characterization of PA gels}

Polyacrylamide (PA) gels of varying stiffness (0.5, 2, and $5 \mathrm{kPa}$ ) were fabricated on 3-APTMS (Sigma) functionalized glass coverslips by combining $40 \%$ acrylamide and $2 \%$ bis-acrylamide in specific ratios as described previously [81]. Gels were crosslinked by addition of $10 \%$ ammonium persulfate (APS) (1:100) and tetramethylethylenediamine (TEMED) (1:1000). After UV crosslinking the gels with Sulfo-SANPAH (Thermo-scientific, Cat \# 22589) in 50 mM HEPES buffer (SRL chemicals, Cat \# 63732), gels were washed with PBS and treated with $25 \mu \mathrm{g} / \mathrm{ml}$ of Type-I collagen (Thermo-scientific, Cat \# A104830) at $4^{\circ} \mathrm{C}$ overnight. Gels were then washed with PBS to remove unbound collagen and incubated with cell culture media for 30 minutes prior to seeding cells.

\section{Conditioned media collection}

For collection of MDA-MB-231 secreted cancer conditioned media (CCM), cancer cells were seeded on PA gels of $0.5,2$, and $5 \mathrm{kPa}$ stiffness at a seeding density of 12000 cells $/ \mathrm{cm}^{2}$. Cells were cultured for $48 \mathrm{hrs}$ in complete media. The collected CCM was centrifuged (1500 rpm, 10 minutes) and filtered through a $0.22 \mu \mathrm{m}$ pore filter prior to use for experiments (Figure 2A). For preparation of CAF-conditioned media (CAFCM), MSC were cultured on different stiffness gels for 7 days in the presence of CCM. After 7 days, differentiated MSCs were washed with PBS and cultured with MSC complete media for next $24 \mathrm{hrs}$ (Fig. 5A). The media 
was then collected, centrifuged and filtered for further use. The collected conditioned media were stored at $-20^{\circ} \mathrm{C}$ or $-80^{\circ} \mathrm{C}$.

\section{Design and fabrication of microfluidic devices}

For studying CCM-induced MSC chemotaxis, we used our previously designed device [38]. For shear experiments, a straight channel $400 \mu \mathrm{m}$ in width having 3 ports was designed (Fig. 6G). Shear stress profile in the zone of interest was simulated using COMSOL MultiPhysics (version 5.2) assuming laminar flow conditions. The device designs were printed on to a transparency mask using a printer with resolution 600 dpi and higher. The design was patterned on a silicon wafer by photolithography with SU-8 (2050) photoresist (MicroChem). PDMS devices were then fabricated by pouring PDMS (Sylgard 184) onto the master, and curing them in the oven. After peeling the PDMS substrates, all ports were punched using a $3 \mathrm{~mm}$ biopsy punch. Finally, devices were fabricated by bonding the PDMS devices to glass.

\section{Cell experiments}

For MSC chemotaxis experiments, $15 \mu \mathrm{l}$ media containing 120000 MSCs (prestained with Hoechst 33342) were mixed with different concentrations of $3 \mathrm{D}$ collagen $(1,2$ and $3 \mathrm{mg} / \mathrm{ml})$ and introduced through one of the channel inlets. Media containing only collagen was introduced through the other inlet. After allowing MSCs to adhere onto the 3D collagen gel ( $\sim 6 \mathrm{hrs}$ ), stiffness-modulated CCM from was introduced as a chemokine at the opposite channel inlet. For measuring cell motility, time-lapse microscopy was performed for 12 hrs using an inverted microscope equipped with an onstage incubator (Evos FL Auto, Life Technologies). Images were acquired every 15 minutes. Cell motility was quantified using the manual cell tracker plugin in ImageJ $(\mathrm{NIH})$.

For differentiation experiments, MSCs were seeded on PA gels at a density of 800 cells $/ \mathrm{cm}^{2}$. After 12 hrs of seeding, CCM collected from $0.5,2$, and $5 \mathrm{kPa}$ stiffness were added onto the respective gels in 1:1 ratio (i.e., CCM: fresh media $=1: 1$ ) for a duration of 7 days. This combined media was replenished after every 48 hrs. For expression profiling of CAF-associated markers, integrins and MMPs, quantitative real time PCR was performed as described elsewhere [82]. Total RNA was isolated using RNeasy Mini Kit (Qiagen, Cat \# 74104). $1 \mu \mathrm{g}$ of total RNA was used in cDNA synthesis using ProtoScript II Reverse Transcription kit (NEB, Cat \# e6560) as per manufacturer's instructions. Primer details are provided in Supp. Table 1.

For immunostaining, MSCs were fixed with ice-cold $4 \%$ paraformaldehyde ( $\mathrm{pH} 7$ ) in the presence of permeabilization buffer (0.5\% TritonX-100) for $1 \mathrm{~min}$ to remove soluble cytoplasmic proteins. After fixation, cells were washed with cytoskeleton stabilizing buffer (CSB) (60mM PIPES, 27 mM HEPES, 10mM EGTA, 4mM magnesium sulphate heptahydrate, $\mathrm{pH} 7$ ) three times, and then incubated with blocking buffer (2\%BSA) for $30 \mathrm{~min}$ at $4^{\circ} \mathrm{C}$. Cells were then incubated with anti- $\alpha-S M A$ antibody (mouse monoclonal, Sigma, Cat\# A2547, 1:400 dilution) and anti-LMNA antibody (mouse monoclonal, Sigma, Cat\# Ab8980, 1:400 dilution), anti-pMLC (rabbit, CST, cat\# 3671S, , 1:400 dilution), anti-Stro-1 (mouse, abcam, cat\# ab102969, 1:400 di- 
bioRxiv preprint doi: https://doi.org/10.1101/2021.01.12.426460; this version posted January $13,2021$. The copyright holder for this preprint

(which was not certified by peer review) is the author/funder, who has granted bioRxiv a license to display the preprint in perpetuity. It is made available under aCC-BY-NC-ND 4.0 International license.

lution). anti-CD 44 (mouse, Novus, cat\# 8E2F3, 1:400 dilution), anti-ITGB1 (rabbit, abcam, cat\# ab183666, 1:400 dilution) overnight at $4^{\circ} \mathrm{C}$ diluted in blocking buffer. The following day, cells were washed with CSB thrice and then incubated with respective secondary antibody (Life Technologies, 1:1000 dilution), Phalloidin (Life Technologies, 1:400 dilution) and Hoechst 33342 (Life Technologies, 1:1000 dilution) for 2 hrs at room temperature (RT). Cells were imaged using laser-scanning confocal microscope (LSM 710, Zeiss 40x magnification), Olympus inverted fluorescence microscope(40x,60x magnification) or Zeiss Spinning disc confocal microscope (20x magnification). Intensity of cells were quantified for multiple cells across 3 independent experiments for each condition using ImageJ (NIH).

For cell proliferation measurements, MSCs cultured on PA gels in the presence and absence of CCM were imaged (15 random images) every alternate day for 7 days. Population doubling was calculated by dividing the average number of cells per frame on a given day by the average number of cells per frame on Day 1. Cell spreading area was determined by manually drawing the outline of individual cells using the polygonal tool in Image J (NIH).

For traction force microscopy (TFM) measurements, $5 \mathrm{kPa}$ gels were prepared on $22 \mathrm{X} 22 \mathrm{~mm}^{2}$ coverslips. Once the gels solidified, a thin layer of $25 \mu \mathrm{l}$ of $5 \mathrm{kPa}$ gel with $1 \mu \mathrm{m}$ rhodamine fluorescent beads (Fluka, 1:50 dilution) was prepared on previously prepared gel. ECM coating was performed as described in previous section. After $24 \mathrm{hr}$ of cell seeding, the cells were imaged at selected location for phase contrast and Red Flurescence Protein (RFP). Cells were then lysed using Triton-X 100 without moving the plate containing the gels and RFP images were again captured. For calculating the magnitude of traction forces, the Matlab code was used from J. P. Butler [83].

For collagen degradation assays, gels were prepared and coated with DQ collagen overnight at $4^{\circ} \mathrm{C}$. After culturing MSCs/CAFs on these substrates for $48 \mathrm{hrs}$, cells were fixed using 4\% PFA, and then stained with Phalloidin and Hoechst 33342, respectively. Fluorescence images (degraded collagen: green, F-actin: red, and nucleus: blue) were captured at 60x magnification. Integrated intensity of degraded collagen per cell was quantified using NIH ImageJ and normalized with respect to that of D1 MSCs.

\section{Spheroid Experiments}

MSCs cultured with CCM on $2 \mathrm{kPa}$ and $5 \mathrm{kPa}$ gels were detached using TrypleE, pelleted and resuspended in media. Cancer cells from tissue culture plastic (TCP) dishes were also trypsinized and re-suspended in media. While homospheroids were generated using MDA-MB-231 cells alone $\left(\mathrm{S}_{M}\right)$, heterospheroids were generated by mixing $2 \mathrm{kPa} / 5 \mathrm{kPa}$ CAFs and cancer cells in a 1:1 ratio $\left(\mathrm{S}_{M F 2}\right.$ and $\mathrm{S}_{M F 5}$, respectively) (Fig. $\left.5 \mathrm{~F}\right)$. Single spheroids were generated using hanging drop $(15 \mu \mathrm{l})$ method by incubating 4000 cells in media containing $6.25 \mu \mathrm{g} / \mathrm{ml}$ of rat-tail collagen I (Thermoscientific, Cat \# A104830) at $37^{\circ} \mathrm{C}, 5 \% \mathrm{CO}_{2}$ for $48 \mathrm{hrs}$. Cell viability within spheroids was assessed by carrying out live/dead staining using Calcein AM (Life Technologies, Cat \# C3099) and propidium iodide (PI, Himedia, Cat \# TC-252). For spheroid invasion experiments, spheroids were embedded in $1.5 \mathrm{mg} / \mathrm{ml}$ 3D collagen gels by mixing collagen with 10X PBS (phosphate buffered saline) 
and DMEM at $4^{\circ} \mathrm{C}$ and kept in incubator for collagen gel formation. After 30 minutes, wells were flooded with media. Images were captured at different time points starting from $t=0 \mathrm{hrs}$ to $t=7$ days. The extent of invasion was quantified and normalized with respect to invasion of MDA-MB-231 spheroids (i.e., $\mathrm{S}_{M}$ ). To assess the spatial position of CAFs in heterospheroids, CAFs were stained with CellTracker red (Thermoscientific, Cat \# C34552) and MDA-MB-231 cells with CellTracker green (Thermoscientific, Cat \# C2925). Spatial distribution of CAFs/cancer cells was observed 48 hrs after implanting spheroids in collagen gels.

For adhesion experiments, spheroids were seeded on fibronectin $(\mathrm{FN})$ coated glass coverslips. Images were taken at $t=0$ to count the number of spheroids seeded and kept for $2 \mathrm{hrs}$ in incubator. Coverslips were washed with PBS after $t=2 \mathrm{hrs}$ to remove non-adherent/weakly-attached spheroids, and then imaged to count the remaining spheroids. Percentage spheroid attachment was calculated across all conditions. For estimating the strength of adhesion, trypsin de-adhesion experiments were performed wherein after 2 hrs of adhesion onto Fn-coated coverslips, spheroids were incubated with Trypsin-EDTA and imaged at 10 sec intervals till the spheroids start to detach, i.e., $t=\tau^{*}[84,85]$. The time required for detachment, $\tau_{\text {detach }}=\left(\tau^{*}-120\right)$ mins, corresponds to the time when there is a visible shift observed in the centroid position of the spheroid as seen in the pseudocolor images (pink and blue) in Fig. 6F.

For shear experiments, PDMS devices designed for shear experiments were bonded to glass using plasma oxidation, and were coated with FN overnight at $4^{\circ} \mathrm{C}$. Next day, after removing unbound FN with PBS wash, device was loaded with media prior to spheroid seeding. Spheroids were seeded in the middle well and allowed to adhere for 10-12 hrs. Subsequently, media from inlet was flown at a rate of $400 \mu \mathrm{l} / \mathrm{min}$. $\tau_{\text {shear }}$ corresponds to the time duration for which individual spheroids remained attached under shear. This was determined based on change in the spheroid position before and after flow was started. The position of individual spheroids is shown in pseudocolor (pink and blue) in Fig. 6I.

\section{Statistical analysis}

All statistical analysis was performed using Origin 9.1 with $p<0.05$ considered to be statistically significant. Based on the normality of data assessed using Kolmogorov-Smirnov normality test, one-way ANOVA/ twoway ANOVA was performed to assess statistical significance, and Fisher post-hoc test was used to compare the means.

\section{Conflict of Interest}

Authors declare no conflict of interest. 


\section{Acknowledgements} available under aCC-BY-NC-ND 4.0 International license.

The authors thank Centre for Nanoelectronics, IIT Bombay for providing lithography facility and IRCC for Confocal (LSM \& Spinning Disc) and AFM Central Facilities. Authors acknowledge financial support from Department of Biotechnology (Govt. of India, Grant \# BT/PR7741/MED/32/275/2013) and Department of Science and Technology (Govt. of India, Grant \# DST/SJF/LSA-01/2016-17). NS was supported by the Inspire Fellowship from the Department of Science and Technology (Govt. of India, DST/INSPIRE Fellowship/2013/1033).

\section{Author Contributions}

Conceptualization: NS, HZ and SS; Methodology: NS, GB SJ, HZ and SS; Experimental analysis: NS; RNAseq analysis: GB and HZ; Writing: NS, HZ and SS.

\section{References}

[1] Koji Shindo, Shinichi Aishima, Kenoki Ohuchida, Kenji Fujiwara, Minoru Fujino, Yusuke Mizuuchi, Masami Hattori, Kazuhiro Mizumoto, Masao Tanaka, and Yoshinao Oda. Podoplanin expression in cancer-associated fibroblasts enhances tumor progression of invasive ductal carcinoma of the pancreas. Molecular Cancer, 12(1):168, dec 2013.

[2] Rosa F. Hwang, Todd Moore, Thiruvengadam Arumugam, Vijaya Ramachandran, Keith D. Amos, Armando Rivera, Baoan Ji, Douglas B. Evans, and Craig D. Logsdon. Cancer-associated stromal fibroblasts promote pancreatic tumor progression. Cancer Research, 68(3):918-926, feb 2008.

[3] Begum Erdogan and Donna J. Webb. Cancer-associated fibroblasts modulate growth factor signaling and extracellular matrix remodeling to regulate tumor metastasis. Biochemical Society Transactions, 45(1):229-236, 2017.

[4] Alakesh Das, Melissa Monteiro, Amlan Barai, Sandeep Kumar, and Shamik Sen. MMP proteolytic activity regulates cancer invasiveness by modulating integrins. Scientific Reports, 7(1):1-13, dec 2017.

[5] James J. Tomasek, Giulio Gabbiani, Boris Hinz, Christine Chaponnier, and Robert A. Brown. Myofibroblasts and mechano: Regulation of connective tissue remodelling, 2002.

[6] Pravin J. Mishra, Prasun J. Mishra, Rita Humeniuk, Daniel J. Medina, Gabriela Alexe, Jill P. Mesirov, Sridhar Ganesan, John W. Glod, and Debabrata Banerjee. Carcinoma-associated fibroblast-like differentiation of human mesenchymal stem cells. Cancer Research, 68(11):4331-4339, 2008.

[7] Shicheng Su, Jianing Chen, Herui Yao, Jiang Liu, Shubin Yu, Liyan Lao, Minghui Wang, Manli Luo, Yue Xing, Fei Chen, Di Huang, Jinghua Zhao, Linbin Yang, Dan Liao, Fengxi Su, Mengfeng Li, Qiang Liu, and Erwei Song. CD10+GPR77+ Cancer-Associated Fibroblasts Promote Cancer Formation and Chemoresistance by Sustaining Cancer Stemness. Cell, 172(4):841-856.e16, feb 2018. 
bioRxiv preprint doi: https://doi.org/10.1101/2021.01.12.426460; this version posted January $13,2021$. The copyright holder for this preprint (which was not certified by peer review) is the author/funder, who has granted bioRxiv a license to display the preprint in perpetuity. It is made available under aCC-BY-NC-ND 4.0 International license.

[8] Ana Costa, Yann Kieffer, Alix Scholer-Dahirel, Floriane Pelon, Brigitte Bourachot, Melissa Cardon, Philemon Sirven, Ilaria Magagna, Laetitia Fuhrmann, Charles Bernard, Claire Bonneau, Maria Kondratova, Inna Kuperstein, Andrei Zinovyev, Anne Marie Givel, Maria Carla Parrini, Vassili Soumelis, Anne Vincent-Salomon, and Fatima Mechta-Grigoriou. Fibroblast Heterogeneity and Immunosuppressive Environment in Human Breast Cancer. Cancer Cell, 33(3):463-479.e10, mar 2018.

[9] Antoine E. Karnoub, Ajeeta B. Dash, Annie P. Vo, Andrew Sullivan, Mary W. Brooks, George W. Bell, Andrea L. Richardson, Kornelia Polyak, Ross Tubo, and Robert A. Weinberg. Mesenchymal stem cells within tumour stroma promote breast cancer metastasis. Nature, 449(7162):557-563, oct 2007.

[10] C. E. Weber, A. N. Kothari, P. Y. Wai, N. Y. Li, J. Driver, M. A.C. Zapf, C. A. Franzen, G. N. Gupta, C. Osipo, A. Zlobin, W. K. Syn, J. Zhang, P. C. Kuo, and Z. Mi. Osteopontin mediates an MZF1-TGF- $\beta 1$-dependent transformation of mesenchymal stem cells into cancer-associated fibroblasts in breast cancer. Oncogene, 34(37):4821-4833, 2015.

[11] Younghun Jung, Jin Koo Kim, Yusuke Shiozawa, Jingcheng Wang, Anjali Mishra, Jeena Joseph, Janice E. Berry, Samantha McGee, Eunsohl Lee, Hongli Sun, Jianhua Wang, Taocong Jin, Honglai Zhang, Jinlu Dai, Paul H. Krebsbach, Evan T. Keller, Kenneth J. Pienta, and Russell S. Taichman. Recruitment of mesenchymal stem cells into prostate tumours promotes metastasis. Nature Communications, 4(1):1-11, apr 2013.

[12] Naoki Shinojima, Anwar Hossain, Tatsuya Takezaki, Juan Fueyo, Joy Gumin, Feng Gao, Felix Nwajei, Frank C. Marini, Michael Andreeff, Jun Ichi Kuratsu, and Frederick F. Lang. TGF- $\beta$ mediates homing of bone marrow-derived human mesenchymal stem cells to glioma stem cells. Cancer Research, 73(7):23332344, apr 2013.

[13] Hui Gao, Waldemar Priebe, John Glod, and Debabrata Banerjee. Activation of signal transducers and activators of transcription 3 and focal adhesion kinase by stromal cell-derived factor 1 is required for migration of human mesenchymal stem cells in response to tumor cell-conditioned medium. Stem Cells, 27(4):857-865, apr 2009.

[14] Christoph Zischek, Hanno Niess, Ivan Ischenko, Claudius Conrad, Ralf Huss, Karl Walter Jauch, Peter J. Nelson, and Christiane Bruns. Targeting tumor stroma using engineered mesenchymal stem cells reduces the growth of pancreatic carcinoma. Annals of Surgery, 250(5):747-752, nov 2009.

[15] Shyam A. Patel, Justin R. Meyer, Steven J. Greco, Kelly E. Corcoran, Margarette Bryan, and Pranela Rameshwar. Mesenchymal Stem Cells Protect Breast Cancer Cells through Regulatory T Cells: Role of Mesenchymal Stem Cell-Derived TGF- $\beta$. The Journal of Immunology, 184(10):5885-5894, may 2010.

[16] Michael R. Loebinger, Panagiotis G. Kyrtatos, Mark Turmaine, Anthony N. Price, Quentin Pankhurst, Mark F. Lythgoe, and Sam M. Janes. Magnetic resonance imaging of mesenchymal stem cells homing to pulmonary metastases using biocompatible magnetic nanoparticles. Cancer Research, 69(23):8862-8867, $\operatorname{dec} 2009$. 
bioRxiv preprint doi: https://doi.org/10.1101/2021.01.12.426460; this version posted January $13,2021$. The copyright holder for this preprint (which was not certified by peer review) is the author/funder, who has granted bioRxiv a license to display the preprint in perpetuity. It is made available under aCC-BY-NC-ND 4.0 International license.

[17] Makio Ogawa, Amanda C. LaRue, and Christopher J. Drake. Hematopoietic origin of fibroblasts/myofibroblasts: Its pathophysiologic implications, nov 2006.

[18] Pallavi Chaturvedi, Daniele M. Gilkes, Carmen Chak Lui Wong, Kshitiz, Weibo Luo, Huafeng Zhang, Hong Wei, Naoharu Takano, Luana Schito, Andre Levchenko, and Gregg L. Semenza. Hypoxia-inducible factor-dependent breast cancer-mesenchymal stem cell bidirectional signaling promotes metastasis. Journal of Clinical Investigation, 123(1):189-205, dec 2012.

[19] Thordur Oskarsson. Extracellular matrix components in breast cancer progression and metastasis. The Breast, 22(S2):S66-S72, aug 2013.

[20] Fernando Calvo, Nil Ege, Araceli Grande-Garcia, Steven Hooper, Robert P. Jenkins, Shahid I. Chaudhry, Kevin Harrington, Peter Williamson, Emad Moeendarbary, Guillaume Charras, and Erik Sahai. Mechanotransduction and YAP-dependent matrix remodelling is required for the generation and maintenance of cancer-associated fibroblasts. Nature Cell Biology, 15(6):637-646, jun 2013.

[21] Hanane Laklai, Yekaterina A. Miroshnikova, Michael W. Pickup, Eric A. Collisson, Grace E. Kim, Alex S. Barrett, Ryan C. Hill, Johnathon N. Lakins, David D. Schlaepfer, Janna K. Mouw, Valerie S. LeBleu, Nilotpal Roy, Sergey V. Novitskiy, Julia S. Johansen, Valeria Poli, Raghu Kalluri, Christine A. IacobuzioDonahue, Laura D. Wood, Matthias Hebrok, Kirk Hansen, Harold L. Moses, and Valerie M. Weaver. Genotype tunes pancreatic ductal adenocarcinoma tissue tension to induce matricellular fibrosis and tumor progression. Nature Medicine, 22(5):497-505, may 2016.

[22] Adam J. Engler, Shamik Sen, H. Lee Sweeney, and Dennis E. Discher. Matrix Elasticity Directs Stem Cell Lineage Specification. Cell, 126(4):677-689, aug 2006.

[23] Seiichiro Ishihara, David R. Inman, Wan Ju Li, Suzanne M. Ponik, and Patricia J. Keely. Mechano-signal transduction in mesenchymal stem cells induces prosaposin secretion to drive the proliferation of breast cancer cells. Cancer Research, 77(22):6179-6189, 2017.

[24] Mihriban Karaayvaz, Simona Cristea, Shawn M. Gillespie, Anoop P. Patel, Ravindra Mylvaganam, Christina C. Luo, Michelle C. Specht, Bradley E. Bernstein, Franziska Michor, and Leif W. Ellisen. Unravelling subclonal heterogeneity and aggressive disease states in TNBC through single-cell RNA-seq. Nature Communications, 9(1), dec 2018.

[25] Ana C. Garrido-Castro, Nancy U. Lin, and Kornelia Polyak. Insights into molecular classifications of triple-negative breast cancer: Improving patient selection for treatment, 2019.

[26] Woosung Chung, Hye Hyeon Eum, Hae Ock Lee, Kyung Min Lee, Han Byoel Lee, Kyu Tae Kim, Han Suk Ryu, Sangmin Kim, Jeong Eon Lee, Yeon Hee Park, Zhengyan Kan, Wonshik Han, and Woong Yang Park. Single-cell RNA-seq enables comprehensive tumour and immune cell profiling in primary breast cancer. Nature Communications, 8, may 2017.

[27] Rahul Satija, Jeffrey A. Farrell, David Gennert, Alexander F. Schier, and Aviv Regev. Spatial reconstruction of single-cell gene expression data. Nature Biotechnology, 33(5):495-502, may 2015. 
bioRxiv preprint doi: https://doi.org/10.1101/2021.01.12.426460; this version posted January $13,2021$. The copyright holder for this preprint (which was not certified by peer review) is the author/funder, who has granted bioRxiv a license to display the preprint in perpetuity. It is made available under aCC-BY-NC-ND 4.0 International license.

[28] Masoud Maleki, Farideh Ghanbarvand, Mohammad Reza Behvarz, Mehri Ejtemaei, and Elham Ghadirkhomi. Comparison of mesenchymal stem cell markers in multiple human adult stem cells. International Journal of Stem Cells, 7(2):118-126, 2014.

[29] Eo Jin Kim, Sang Gyo Seo, Hyuk Soo Shin, Doo Jae Lee, Ji Hye Kim, and Dong Yeon Lee. Plateletderived growth factor receptor-positive pericytic cells of white adipose tissue from critical limb ischemia patients display mesenchymal stem cell-like properties. CiOS Clinics in Orthopedic Surgery, 9(2):239-248, jun 2017.

[30] Beatriz Roson-Burgo, Fermin Sanchez-Guijo, Consuelo Del Cañizo, and Javier De Las Rivas. Insights into the human mesenchymal stromal/stem cell identity through integrative transcriptomic profiling. BMC Genomics, 17(1), nov 2016.

[31] Wasim S. Khan, Adetola B. Adesida, Simon R. Tew, Emma T. Lowe, and Timothy E. Hardingham. Bone marrow-derived mesenchymal stem cells express the pericyte marker 3G5 in culture and show enhanced chondrogenesis in hypoxic conditions. Journal of Orthopaedic Research, 28(6):834-840, jun 2010.

[32] Yi Tang, Lin Liu, Pei Wang, Donglei Chen, Ziqiang Wu, and Chunbo Tang. Periostin promotes migration and osteogenic differentiation of human periodontal ligament mesenchymal stem cells via the Jun aminoterminal kinases (JNK) pathway under inflammatory conditions. Cell Proliferation, 50(6), dec 2017.

[33] Sunny Z Wu, Daniel L Roden, Chenfei Wang, Holly Holliday, Kate Harvey, Aurélie S Cazet, Kendelle J Murphy, Brooke Pereira, Ghamdan Al-Eryani, Nenad Bartonicek, Rui Hou, James R Torpy, Simon Junankar, Chia-Ling Chan, Chuan En Lam, Mun N Hui, Laurence Gluch, Jane Beith, Andrew Parker, Elizabeth Robbins, Davendra Segara, Cindy Mak, Caroline Cooper, Sanjay Warrier, Alistair Forrest, Joseph Powell, Sandra O'Toole, Thomas R Cox, Paul Timpson, Elgene Lim, X Shirley Liu, and Alexander Swarbrick. Stromal cell diversity associated with immune evasion in human triple-negative breast cancer. The EMBO Journal, 39(19), oct 2020.

[34] Junyue Cao, Malte Spielmann, Xiaojie Qiu, Xingfan Huang, Daniel M. Ibrahim, Andrew J. Hill, Fan Zhang, Stefan Mundlos, Lena Christiansen, Frank J. Steemers, Cole Trapnell, and Jay Shendure. The single-cell transcriptional landscape of mammalian organogenesis. Nature, 566(7745):496-502, feb 2019.

[35] Matthew J. Paszek, Nastaran Zahir, Kandice R. Johnson, Johnathon N. Lakins, Gabriela I. Rozenberg, Amit Gefen, Cynthia A. Reinhart-King, Susan S. Margulies, Micah Dembo, David Boettiger, Daniel A. Hammer, and Valerie M. Weaver. Tensional homeostasis and the malignant phenotype. Cancer Cell, $8(3): 241-254$, sep 2005.

[36] Kandice R. Levental, Hongmei Yu, Laura Kass, Johnathon N. Lakins, Mikala Egeblad, Janine T. Erler, Sheri F.T. Fong, Katalin Csiszar, Amato Giaccia, Wolfgang Weninger, Mitsuo Yamauchi, David L. Gasser, and Valerie M. Weaver. Matrix Crosslinking Forces Tumor Progression by Enhancing Integrin Signaling. Cell, 139(5):891-906, nov 2009. 
bioRxiv preprint doi: https://doi.org/10.1101/2021.01.12.426460; this version posted January $13,2021$. The copyright holder for this preprint (which was not certified by peer review) is the author/funder, who has granted bioRxiv a license to display the preprint in perpetuity. It is made available under aCC-BY-NC-ND 4.0 International license.

[37] Hongmei Yu, Janna Kay Mouw, and Valerie M. Weaver. Forcing form and function: Biomechanical regulation of tumor evolution. Trends in Cell Biology, 21(1):47-56, jan 2011.

[38] Neha Saxena, Pankaj Mogha, Silalipi Dash, Abhijit Majumder, Sameer Jadhav, and Shamik Sen. Matrix elasticity regulates mesenchymal stem cell chemotaxis. Journal of Cell Science, page jcs.211391, 2018.

[39] D. Lazard, X. Sastre, M. G. Frid, M. A. Glukhova, J. P. Thiery, and V. E. Koteliansky. Expression of smooth muscle-specific proteins in myoepithelium and stromal myofibroblasts of normal and malignant human breast tissue. Proceedings of the National Academy of Sciences of the United States of America, 90(3):999-1003, 1993.

[40] Anna Labernadie, Takuya Kato, Agustí Brugués, Xavier Serra-Picamal, Stefanie Derzsi, Esther Arwert, Anne Weston, Victor González-Tarragó, Alberto Elosegui-Artola, Lorenzo Albertazzi, Jordi Alcaraz, Pere Roca-Cusachs, Erik Sahai, and Xavier Trepat. A mechanically active heterotypic E-cadherin/N-cadherin adhesion enables fibroblasts to drive cancer cell invasion. Nature Cell Biology, 19(3):224-237, 2017.

[41] Erdem Kucukal, Jane A. Little, and Umut A. Gurkan. Shear dependent red blood cell adhesion in microscale flow. Integrative Biology (United Kingdom), 10(4):194-206, apr 2018.

[42] Barbara Maria Szczerba, Francesc Castro-Giner, Marcus Vetter, Ilona Krol, Sofia Gkountela, Julia Landin, Manuel C. Scheidmann, Cinzia Donato, Ramona Scherrer, Jochen Singer, Christian Beisel, Christian Kurzeder, Viola Heinzelmann-Schwarz, Christoph Rochlitz, Walter Paul Weber, Niko Beerenwinkel, and Nicola Aceto. Neutrophils escort circulating tumour cells to enable cell cycle progression. Nature, 566(7745):553-557, feb 2019.

[43] Xia Liu, Rokana Taftaf, Madoka Kawaguchi, Ya Fang Chang, Wenjing Chen, David Entenberg, Youbin Zhang, Lorenzo Gerratana, Simo Huang, Dhwani B. Patel, Elizabeth Tsui, Valery Adorno-Cruz, Steven M. Chirieleison, Yue Cao, Allison S. Harney, Shivani Patel, Antonia Patsialou, Yang Shen, Stefanie Avril, Hannah L. Gilmore, Justin D. Lathia, Derek W. Abbott, Massimo Cristofanilli, John S. Condeelis, and Huiping Liu. Homophilic CD44 interactions mediate tumor cell aggregation and polyclonal metastasis in patient-derived breast cancer models. Cancer Discovery, 9(1):96-113, 2019.

[44] Ryan T. Davis, Kerrigan Blake, Dennis Ma, Mari B.Ishak Gabra, Grace A. Hernandez, Anh T. Phung, Ying Yang, Dustin Maurer, Austin E.Y.T. Lefebvre, Hamad Alshetaiwi, Zhengtao Xiao, Juan Liu, Jason W. Locasale, Michelle A. Digman, Eric Mjolsness, Mei Kong, Zena Werb, and Devon A. Lawson. Transcriptional diversity and bioenergetic shift in human breast cancer metastasis revealed by single-cell RNA sequencing. Nature Cell Biology, 22(3):310-320, mar 2020.

[45] Tim Stuart, Andrew Butler, Paul Hoffman, Christoph Hafemeister, Efthymia Papalexi, William M. Mauck, Yuhan Hao, Marlon Stoeckius, Peter Smibert, and Rahul Satija. Comprehensive Integration of Single-Cell Data. Cell, 177(7):1888-1902.e21, jun 2019. 
bioRxiv preprint doi: https://doi.org/10.1101/2021.01.12.426460; this version posted January $13,2021$. The copyright holder for this preprint (which was not certified by peer review) is the author/funder, who has granted bioRxiv a license to display the preprint in perpetuity. It is made available under aCC-BY-NC-ND 4.0 International license.

[46] Luke Bu, Hideo Baba, Naoya Yoshida, Keisuke Miyake, Tadahito Yasuda, Tomoyuki Uchihara, Patrick Tan, and Takatsugu Ishimoto. Biological heterogeneity and versatility of cancer-associated fibroblasts in the tumor microenvironment, jun 2019.

[47] Pedro Barcellos-de Souza, Giuseppina Comito, Coral Pons-Segura, Maria Letizia Taddei, Valentina Gori, Valentina Becherucci, Franco Bambi, Francesca Margheri, Anna Laurenzana, Mario Del Rosso, and Paola Chiarugi. Mesenchymal Stem Cells are Recruited and Activated into Carcinoma-Associated Fibroblasts by Prostate Cancer Microenvironment-Derived TGF- $\beta 1$. Stem Cells, 34(10):2536-2547, oct 2016.

[48] Panseon Ko, Daehwan Kim, Eunae You, Jangho Jung, Somi Oh, Jaehyun Kim, Kwang Ho Lee, and Sangmyung Rhee. Extracellular Matrix Rigidity-dependent Sphingosine-1-phosphate Secretion Regulates Metastatic Cancer Cell Invasion and Adhesion. Scientific Reports, 6(1):21564, feb 2016.

[49] Yaxian Kong, Hong Wang, Tao Lin, and Shuling Wang. Sphingosine-1-phosphate/s1p receptors signaling modulates cell migration in human bone marrow-derived mesenchymal stem cells. Mediators of Inflammation, 2014, 2014.

[50] Vincent Bernard, Alexander Semaan, Jonathan Huang, F. Anthony San Lucas, Feven C. Mulu, Bret M. Stephens, Paola A. Guerrero, Yanqing Huang, Jun Zhao, Nabiollah Kamyabi, Subrata Sen, Paul A. Scheet, Cullen M. Taniguchi, Michael P. Kim, Ching Wei Tzeng, Matthew H. Katz, Aatur D. Singhi, Anirban Maitra, and Hector A. Alvarez. Single-cell transcriptomics of pancreatic cancer precursors demonstrates epithelial and microenvironmental heterogeneity as an early event in neoplastic progression. Clinical Cancer Research, 25(7):2194-2205, apr 2019.

[51] Diana Avery, Priya Govindaraju, Michele Jacob, Leslie Todd, James Monslow, and Ellen Puré. Extracellular matrix directs phenotypic heterogeneity of activated fibroblasts. Matrix Biology, 67:90-106, 2018.

[52] Ming Hui Fan, Qiang Zhu, Hui Hua Li, Hyun Jeong Ra, Sonali Majumdar, Dexter L. Gulick, Jacob A. Jerome, Daniel H. Madsen, Melpo Christofidou-Solomidou, David W. Speicher, William W. Bachovchin, Carol Feghali-Bostwick, and Ellen Puré. Fibroblast activation protein (FAP) accelerates collagen degradation and clearance from lungs in mice. Journal of Biological Chemistry, 291(15):8070-8089, apr 2016.

[53] Jun Jia, Tracey A. Martin, Lin Ye, and Wen G. Jiang. FAP- $\alpha$ (Fibroblast activation protein- $\alpha$ ) is involved in the control of human breast cancer cell line growth and motility via the FAK pathway. $B M C$ Cell Biology, 15(1), may 2014.

[54] Tomoya Kawase, Yumiko Yasui, Sohji Nishina, Yuichi Hara, Izumi Yanatori, Yasuyuki Tomiyama, Yoshihiro Nakashima, Koji Yoshida, Fumio Kishi, Masafumi Nakamura, and Keisuke Hino. Fibroblast activation protein-a $\alpha$-expressing fibroblasts promote the progression of pancreatic ductal adenocarcinoma. BMC Gastroenterology, 15(1), sep 2015.

[55] Peio Errarte, Rosa Guarch, Rafael Pulido, Lorena Blanco, Caroline E. Nunes-Xavier, Maider Beitia, Javier Gil, Javier C. Angulo, José I. Lopez, and Gorka Larrinaga. The expression of fibroblast activation 
bioRxiv preprint doi: https://doi.org/10.1101/2021.01.12.426460; this version posted January $13,2021$. The copyright holder for this preprint

(which was not certified by peer review) is the author/funder, who has granted bioRxiv a license to display the preprint in perpetuity. It is made available under aCC-BY-NC-ND 4.0 International license.

protein in clear cell renal cell carcinomas is associated with synchronous lymph node metastases. PLoS ONE, 11(12), dec 2016.

[56] Angelo L. Grauel, Beverly Nguyen, David Ruddy, Tyler Laszewski, Stephanie Schwartz, Jonathan Chang, Julie Chen, Michelle Piquet, Marc Pelletier, Zheng Yan, Nathaniel D. Kirkpatrick, Jincheng Wu, Antoine DeWeck, Markus Riester, Matt Hims, Felipe Correa Geyer, Joel Wagner, Kenzie MacIsaac, James Deeds, Rohan Diwanji, Pushpa Jayaraman, Yenyen Yu, Quincey Simmons, Shaobu Weng, Alina Raza, Brian Minie, Mirek Dostalek, Pavitra Chikkegowda, Vera Ruda, Oleg Iartchouk, Naiyan Chen, Raphael Thierry, Joseph Zhou, Iulian Pruteanu-Malinici, Claire Fabre, Jeffrey A. Engelman, Glenn Dranoff, and Viviana Cremasco. TGF $\beta$-blockade uncovers stromal plasticity in tumors by revealing the existence of a subset of interferon-licensed fibroblasts. Nature Communications, 11(1), dec 2020.

[57] Johan Malmstrom, Henrik Linberg, Claes Lindberg, Charlotte Bratt, Elisabet Wieslander, Eva Lena Delander, Bengt Särnstrand, Jorge S. Burns, Peter Mose-Larsen, Stephen Fey, and György Marko-Varga. Transforming growth factor- $\beta 1$ specificically induce proteins involved in the myofibroblast contractile apparatus, may 2004.

[58] Tobias Meyer-Ter-Vehn, Sonja Sieprath, Barbara Katzenberger, Susanne Gebhardt, Franz Grehn, and Günther Schlunck. Contractility as a prerequisite for TGF- $\beta$-induced myofibroblast transdifferentiation in human tenon fibroblasts. Investigative Ophthalmology and Visual Science, 47(11):4895-4904, nov 2006.

[59] Kai Hui Sun, Yongen Chang, Nilgun I. Reed, and Dean Sheppard. $\alpha$-smooth muscle actin is an inconsistent marker of fibroblasts responsible for force-dependent TGF $\beta$ activation or collagen production across multiple models of organ fibrosis. American Journal of Physiology - Lung Cellular and Molecular Physiology, 310(9):L824-L836, may 2016.

[60] Pierre Jean Wipff, Daniel B. Rifkin, Jean Jacques Meister, and Boris Hinz. Myofibroblast contraction activates latent TGF- $\beta 1$ from the extracellular matrix. Journal of Cell Biology, 179(6):1311-1323, dec 2007.

[61] Daniel Öhlund, Abram Handly-Santana, Giulia Biffi, Ela Elyada, Ana S. Almeida, Mariano Ponz-Sarvise, Vincenzo Corbo, Tobiloba E. Oni, Stephen A. Hearn, Eun Jung Lee, Iok In Christine Chio, Chang Il Hwang, Hervé Tiriac, Lindsey A. Baker, Dannielle D. Engle, Christine Feig, Anne Kultti, Mikala Egeblad, Douglas T. Fearon, James M. Crawford, Hans Clevers, Youngkyu Park, and David A. Tuveson. Distinct populations of inflammatory fibroblasts and myofibroblasts in pancreatic cancer. The Journal of experimental medicine, 214(3):579-596, mar 2017.

[62] Giulia Biffi, Tobiloba E. Oni, Benjamin Spielman, Yuan Hao, Ela Elyada, Youngkyu Park, Jonathan Preall, and David A. Tuveson. Il1-induced Jak/STAT signaling is antagonized by TGF $\beta$ to shape CAF heterogeneity in pancreatic ductal adenocarcinoma. Cancer Discovery, 9(2):282-301, feb 2019.

[63] Micol Eleonora Fiori, Simone Di Franco, Lidia Villanova, Paola Bianca, Giorgio Stassi, and Ruggero De Maria. Cancer-associated fibroblasts as abettors of tumor progression at the crossroads of EMT and therapy resistance, mar 2019. 
bioRxiv preprint doi: https://doi.org/10.1101/2021.01.12.426460; this version posted January 13, 2021. The copyright holder for this preprint (which was not certified by peer review) is the author/funder, who has granted bioRxiv a license to display the preprint in perpetuity. It is made available under aCC-BY-NC-ND 4.0 International license.

[64] Lee Ann Jolly, Sergey Novitskiy, Phillip Owens, Nicole Massoll, Nikki Cheng, Wei Fang, Harold L. Moses, and Aime T. Franco. Fibroblast-mediated collagen remodeling within the tumor microenvironment facilitates progression of thyroid cancers driven by brafv600e and pten loss. Cancer Research, 76(7):18041813, apr 2016.

[65] Daniela Pankova, Yulong Chen, Masahiko Terajima, Mark J. Schliekelman, Brandi N. Baird, Monica Fahrenholtz, Li Sun, Bartley J. Gill, Tegy J. Vadakkan, Min P. Kim, Young Ho Ahn, Jonathon D. Roybal, Xin Liu, Edwin Roger Parra Cuentas, Jaime Rodriguez, Ignacio I. Wistuba, Chad J. Creighton, Don L. Gibbons, John M. Hicks, Mary E. Dickinson, Jennifer L. West, K. Jane Grande-Allen, Samir M. Hanash, Mitsuo Yamauchi, and Jonathan M. Kurie. Cancer-associated fibroblasts induce a collagen cross-link switch in tumor stroma. Molecular Cancer Research, 14(3):287-295, mar 2016.

[66] Yonglei Liu, Conghui Yu, Yonggang Wu, Xiangjun Sun, Quanping Su, Cuiping You, and Hongwu Xin. CD44+ fibroblasts increases breast cancer cell survival and drug resistance via IGF2BP3-CD44-IGF2 signalling. Journal of Cellular and Molecular Medicine, 21(9):1979-1988, sep 2017.

[67] Nicola Aceto, Aditya Bardia, David T. Miyamoto, Maria C. Donaldson, Ben S. Wittner, Joel A. Spencer, Min Yu, Adam Pely, Amanda Engstrom, Huili Zhu, Brian W. Brannigan, Ravi Kapur, Shannon L. Stott, Toshi Shioda, Sridhar Ramaswamy, David T. Ting, Charles P. Lin, Mehmet Toner, Daniel A. Haber, and Shyamala Maheswaran. Circulating tumor cell clusters are oligoclonal precursors of breast cancer metastasis. Cell, 158(5):1110-1122, 2014.

[68] Dan G. Duda, Annique M.M.J. Duyverman, Mitsutomo Kohno, Matija Snuderl, Ernst J.A. Steller, Dai Fukumura, and Rakesh K. Jain. Malignant cells facilitate lung metastasis by bringing their own soil. Proceedings of the National Academy of Sciences of the United States of America, 107(50):21677-21682, $\operatorname{dec} 2010$.

[69] Zheng Ao, Sanket H. Shah, Leah M. Machlin, Ritesh Parajuli, Philip C. Miller, Siddarth Rawal, Anthony J. Williams, Richard J. Cote, Marc E. Lippman, Ram H. Datar, and Dorraya El-Ashry. Identification of cancer-associated fibroblasts in circulating blood from patients with metastatic breast cancer. Cancer Research, 75(22):4681-4687, nov 2015.

[70] Martin R. Weber, Masahiko Zuka, Mihaela Lorger, Mario Tschan, Bruce E. Torbett, Andries Zijlstra, James P. Quigley, Karin Staflin, Brian P. Eliceiri, Joseph S. Krueger, Patrizia Marchese, Zaverio M. Ruggeri, and Brunhilde H. Felding. Activated tumor cell integrin $\alpha \mathrm{v} \beta 3$ cooperates with platelets to promote extravasation and metastasis from the blood stream. Thrombosis Research, 140(Suppl 1):S27S36, apr 2016.

[71] Nerymar Ortiz-Otero, Andrea B. Clinch, Jacob Hope, Wenjun Wang, Cynthia A. Reinhart-King, and Michael R. King. Cancer associated fibroblasts confer shear resistance to circulating tumor cells during prostate cancer metastatic progression. Oncotarget, 11(12):1037-1050, jan 2020. 
bioRxiv preprint doi: https://doi.org/10.1101/2021.01.12.426460; this version posted January $13,2021$. The copyright holder for this preprint (which was not certified by peer review) is the author/funder, who has granted bioRxiv a license to display the preprint in perpetuity. It is made available under aCC-BY-NC-ND 4.0 International license.

[72] Rongsheng Zhang, Fan Qi, Shengli Shao, Geng Li, and Yongdong Feng. Human colorectal cancer-derived carcinoma associated fibroblasts promote CD44-mediated adhesion of colorectal cancer cells to endothelial cells by secretion of HGF. Cancer Cell International, 19(1):192, dec 2019.

[73] Erik H.J. Danen, Petra Sonneveld, Cord Brakebusch, Reinhard Fässler, and Arnoud Sonnenberg. The fibronectin-binding integrins $\alpha 5 \beta 1$ and $\alpha \mathrm{v} \beta 3$ differentially modulate RhoA-GTP loading, organization of cell matrix adhesions, and fibronectin fibrillogenesis. Journal of Cell Biology, 159(6):1071-1086, dec 2002.

[74] Naël Osmani, Gautier Follain, María J. García León, Olivier Lefebvre, Ignacio Busnelli, Annabel Larnicol, Sébastien Harlepp, and Jacky G. Goetz. Metastatic Tumor Cells Exploit Their Adhesion Repertoire to Counteract Shear Forces during Intravascular Arrest. Cell Reports, 28(10):2491-2500.e5, 2019.

[75] Don X. Nguyen, Paula D. Bos, and Joan Massagué. Metastasis: From dissemination to organ-specific colonization, apr 2009.

[76] William C. Aird. Phenotypic heterogeneity of the endothelium: I. Structure, function, and mechanisms, feb 2007.

[77] Xiang H.F. Zhang, Xin Jin, Srinivas Malladi, Yilong Zou, Yong H. Wen, Edi Brogi, Marcel Smid, John A. Foekens, and Joan Massagué. Selection of bone metastasis seeds by mesenchymal signals in the primary tumor stroma. Cell, 154(5):1060-1073, 2013.

[78] Juan Ren, Hui Guo, Huili Wu, Tao Tian, Danfeng Dong, Yuelang Zhang, Yanxia Sui, Yong Zhang, Dongli Zhao, Shufeng Wang, Zongfang Li, Xiaozhi Zhang, Rui Liu, Jianshneg Qian, Hongxia Wei, Wenjun Jiang, Ya Liu, and Yi Li. GPER in CAFs regulates hypoxia-driven breast cancer invasion in a CTGF-dependent manner. Oncology Reports, 33(4):1929-1937, apr 2015.

[79] Michal Rajski, Rosanna Zanetti-Dällenbach, Brigitte Vogel, Richard Herrmann, Christoph Rochlitz, and Martin Buess. IGF-I induced genes in stromal fibroblasts predict the clinical outcome of breast and lung cancer patients. BMC Medicine, 8(1):1, jan 2010.

[80] Helen Liapis, Alanna Flath, and Sohei Kitazawa. Integrin $\alpha \mathrm{v} \beta 3$ Expression by Bone-residing Breast Cancer Metastases. Diagnostic Molecular Pathology, 5(2):127-135, 1996.

[81] Justin R. Tse and Adam J. Engler. Preparation of hydrogel substrates with tunable mechanical properties. Current Protocols in Cell Biology, Chapter 10(SUPPL. 47):Unit 10.16, jun 2010.

[82] Kenneth J. Livak and Thomas D. Schmittgen. Analysis of relative gene expression data using real-time quantitative PCR and the 2- $\Delta \Delta \mathrm{CT}$ method. Methods, 25(4):402-408, 2001.

[83] J. P. Butler, I. M. Tolic-Norrelykke, B. Fabry, and J. J. Fredberg. Traction fields, moments, and strain energy that cells exert on their surroundings. AJP: Cell Physiology, 282(3):C595-C605, mar 2002. 
[84] S. S. Soumya, Subodh Kolwankar, Edna George, Santanu K. Basu, Shamik Sen, and Mandar M. Inamdar. Spatial anisotropy and heterogeneity in contractility and adhesion distribution may contribute to cell steering during migration. Applied Physics Letters, 104(8), 2014.

[85] S. S. Soumya, Lakshmi Kavitha Sthanam, Ranjith Padinhateeri, Mandar M. Inamdar, and Shamik Sen. Probing cellular mechanoadaptation using cell-substrate de-adhesion dynamics: Experiments and model. PLoS ONE, 9(9), sep 2014. 
bioRxiv preprint doi: https://doi.org/10.1101/2021.01.12.426460; this version posted January 13,2021 . The copyright holder for this preprint (which was not certified by peer review) is the author/funder, who has granted bioRxiv a license to display the preprint in perpetuity. It is made

Figures \& Figure Legends

A

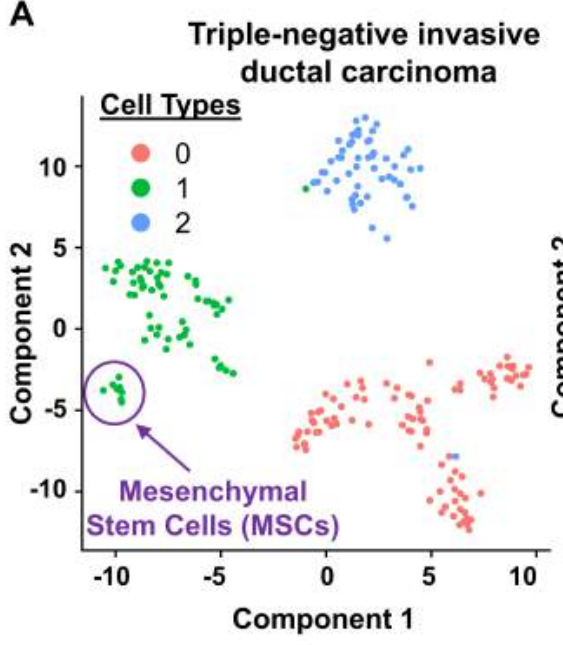

C

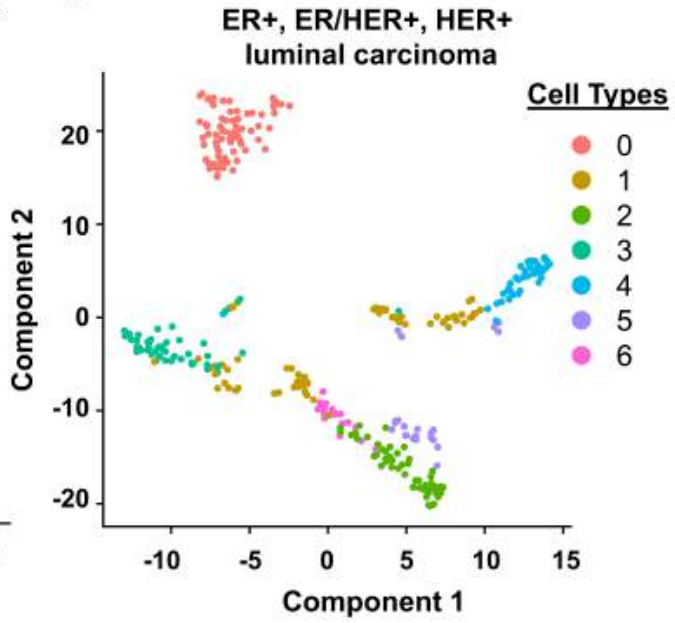

B

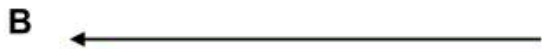

Triple-negative invasive ductal carcinoma

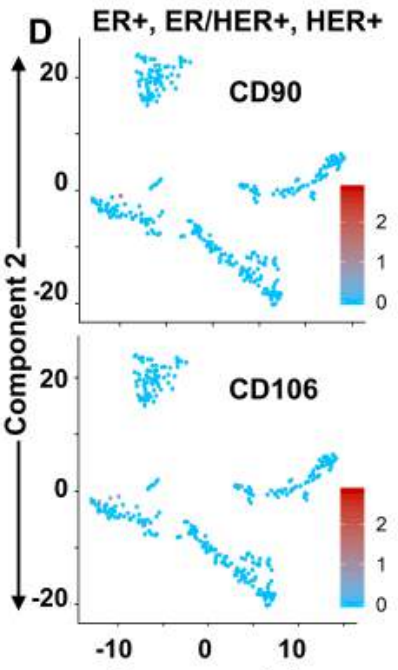

Component 1
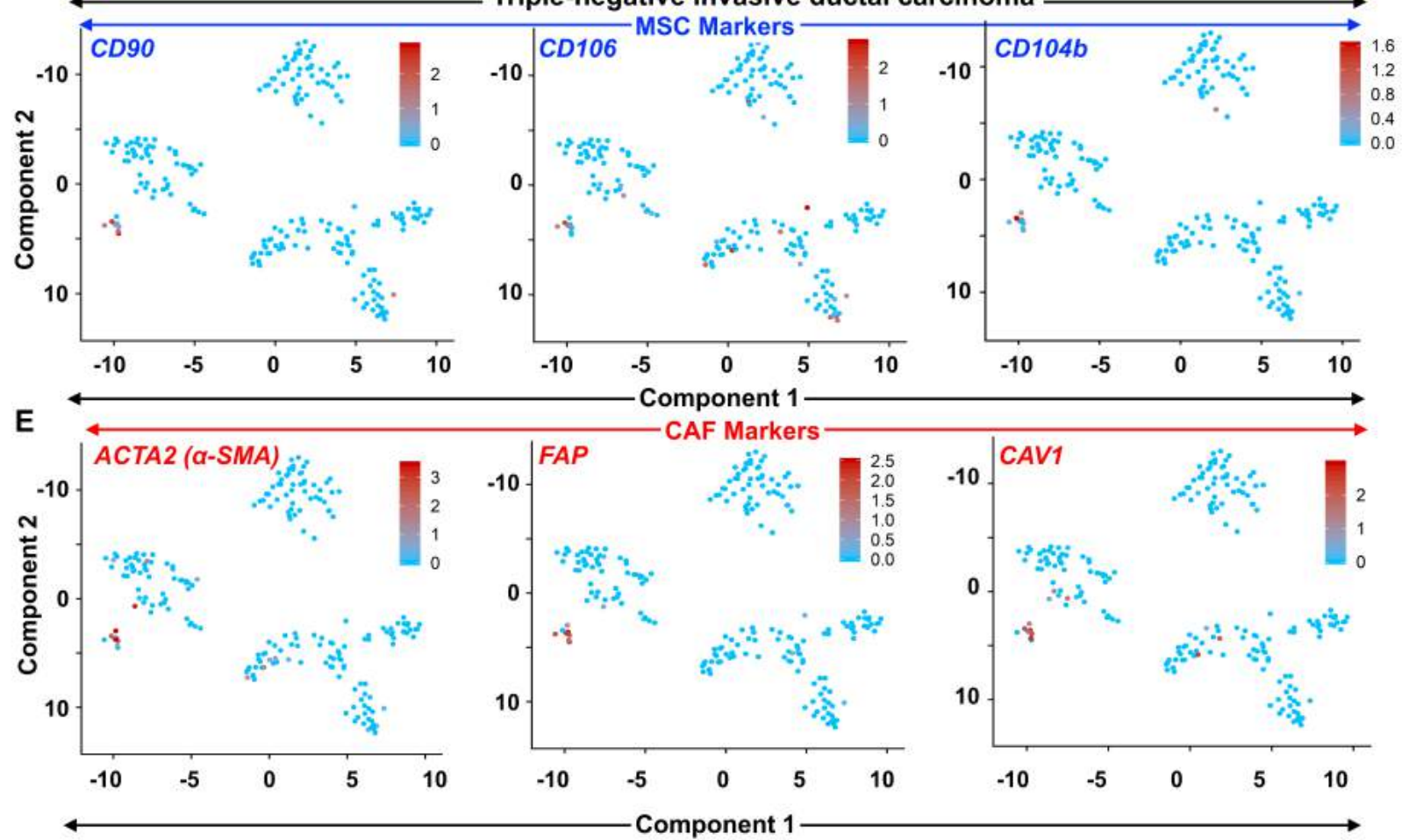

Figure 1: scRNAseq Analysis of TNBC and non-TNBC Data set: A) UMAP plot of cells from triple negative breast cancer (TNBC) patients delineates three cell type clusters, out of which cluster 1 harboured putative mesenchymal stem cells (MSCs). B) The subset of 10 cells in cluster 1 (green) expressed several MSC markers (CD90, CD106. CD104b). C) UMAP plot of cells from non-TNBC patients showing 7 cell type clusters. D) Cells from non-TNBC patients did not express MSC markers. E) The putative MSCs (Cluster 1) were also found to express known CAF markers, including $\alpha-S M A$, FAP, CAV1. 

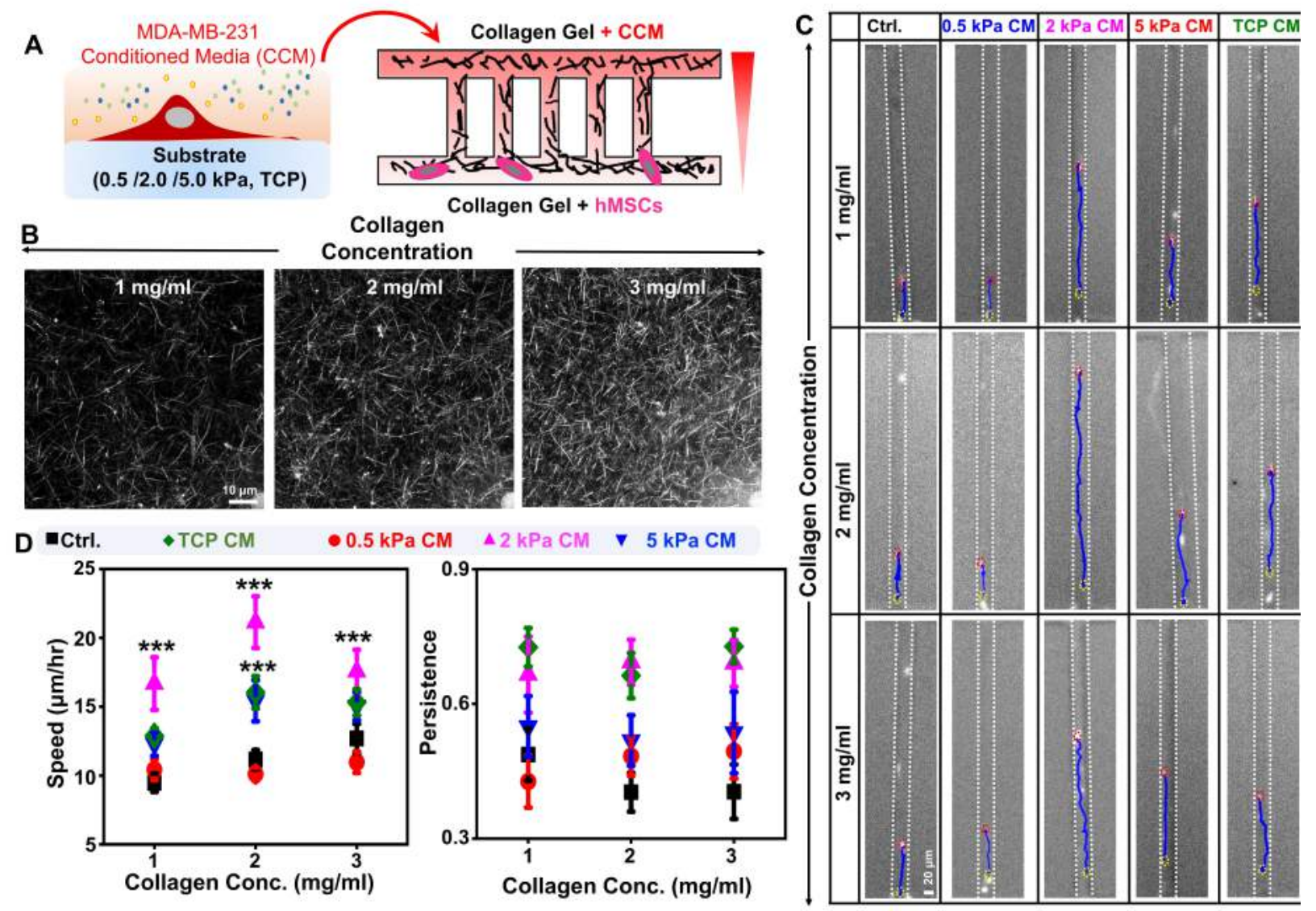

Figure 2: Influence of stiffness-modulated cancer cell conditioned media (CCM) on MSC chemotaxis. A) CCM collected from MDA-MB-231 breast cancer cells cultured on $0.5,2$, and $5 \mathrm{kPa}$ polyacrylamide (PA) gels was used as a chemical cue to study MSC chemotaxis. Chemotaxis was studied within a microfluidic device consisting of two side-channels connected by multiple transverse channels. While MSCs mixed with 3D collagen solution was introduced in the bottom channel, collagen solution was introduced in the top channel. After collagen gel formation at $37^{\circ} \mathrm{C}$, CCM was introduced in the top channel to setup a chemokine gradient within the transverse channels. B) Confocal reflectance images of polymerized collagen at different concentrations $(1,2$, and $3 \mathrm{mg} / \mathrm{ml}$ ). C) Representative trajectories (blue lines) of MSCs migrating through transverse channels in the presence or absence of stiffness-modulated CCM. Cells were tracked by labeling nuclei with Hoechst 33342. Scale bar $=20 \mu \mathrm{m}$ ). D) Quantification of cell speed and persistence of MSCs migrating through transverse channels at varying collagen concentrations in the absence (Ctrl) and presence of CCM ( $n \geq 30$ cells per condition pooled from $N=3$ independent experiments; error bars represent \pm $\mathrm{SEM} ; * * *$ indicates statistical significance $(p<0.001)$ compared to $0.5 \mathrm{kPa} \mathrm{CM}$ condition $)$. 
bioRxiv preprint doi: https://doi.org/10.1101/2021.01.12.426460; this version posted January 13, 2021. The copyright holder for this preprint (which was not certified by peer review) is the author/funder, who has granted bioRxiv a license to display the preprint in perpetuity. It is made available under aCC-BY-NC-ND 4.0 International license.
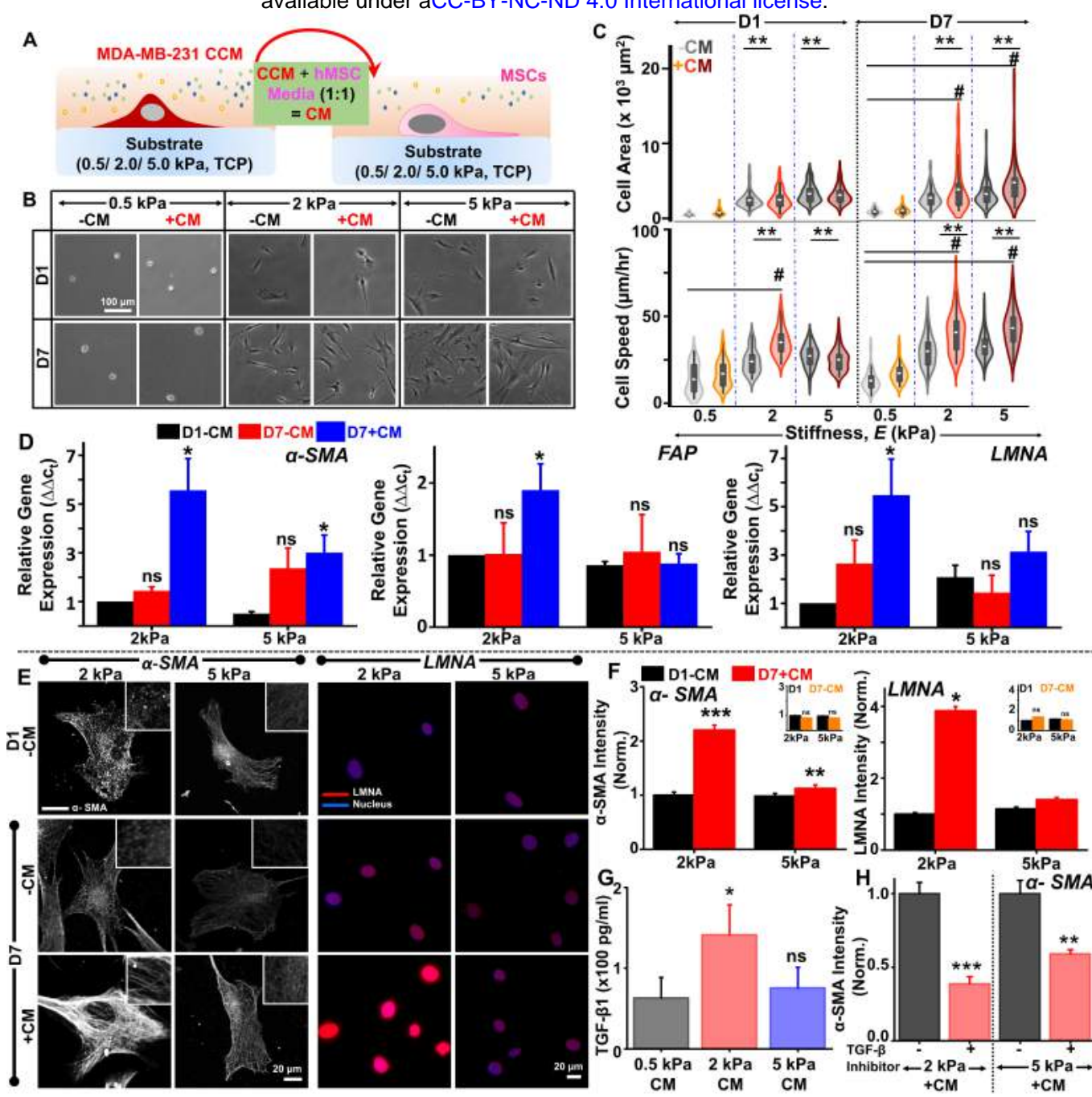

Figure 3: Matrix stiffness regulates MSC differentiation into CAFs: A) Experimental plan for studying the effect of stiffness-modulated CCM on MSC differentiation. MSCs were cultured in conditioned media (CM) generated by mixing stiffness-modulated CCM with MSC complete media in 1:1 ratio. B) Representative images of MSCs cultured on $0.5,2$, and $5 \mathrm{kPa}$ PA gels $\pm \mathrm{CM}$. Images were acquired at Day 1 (D1) and Day 7 (D7). Scale bar $=100 \mu \mathrm{m}$. C) Quantification of MSC spreading and motility at D1 and D7 across different conditions ( $n \geq 50$ cells per condition pooled from $N=3$ independent experiments; error bars represent \pm $\mathrm{SEM}, * *$ signifies statistical significance $(p<0.01)$ w.r.t $0.5 \mathrm{kPa}(-\mathrm{CM})$ condition at D1 and D7 respectively and \# signifies statistical significance $(p<0.05)$ within subgroups). D) Expression profile of CAF markers $(\alpha-S M A, F A P)$ and differentiation marker $(L M N A)$ in MSCs cultured on (2 and 5) kPa gels \pm CM for 7 days $(N=3$ independent experiments; Error bars represent $\pm \mathrm{SEM} ; *$ signifies statistical significance $(p<0.05)$ w.r.t D1-CM condition). E, F) Representative images and intensity quantification of $\alpha-S M A / L M N A$ stained MSCs cultured on (2 and 5) kPa gels $\pm \mathrm{CM}$ at D1 and D7. Scale bar $=20 \mu \mathrm{m}$. For image quantification, integrated intensities were normalized w.r.t $2 \mathrm{kPa},-\mathrm{CM}, \mathrm{D} 1$ condition $(n \geq 50$ cells per condition from $N=3$ independent experiments; error bars represent $\pm \mathrm{SEM}, * * *, * *, *$ indicates statistical significance $(p<$ $0.001, p<0.01, p<0.05$, respectively) compared to D1-CM). Insets show integrated intensities normalized to $2 \mathrm{kPa}$, - CM, D1 condition for cells cultured in the absence of CM on (2 and 5) kPa gels. G) ELISAbased quantification of TGF $\beta$ levels in stiffness-modulated CCM $(N=3$ independent experiments; Error bars represent $\pm \mathrm{SEM} ; *$ signifies statistical significance $(p<0.05)$ w.r.t D1-CM condition $)$. Error bars represent \pm SEM. H) $\alpha$-SMA levels in MSCs cultured on (2 and 5) kPa gels \pm TGF $\beta$ neutralizing antibody for 7 days in the presence of CM ( $n \geq 90$ cells per condition pooled from $N=3$ independent experiments; error bars represent $\pm \mathrm{SEM}, * * *, * *$ indicates statistical significance $(p<0.001, p<0.01$, respectively) compared to no inhibitor condition). 


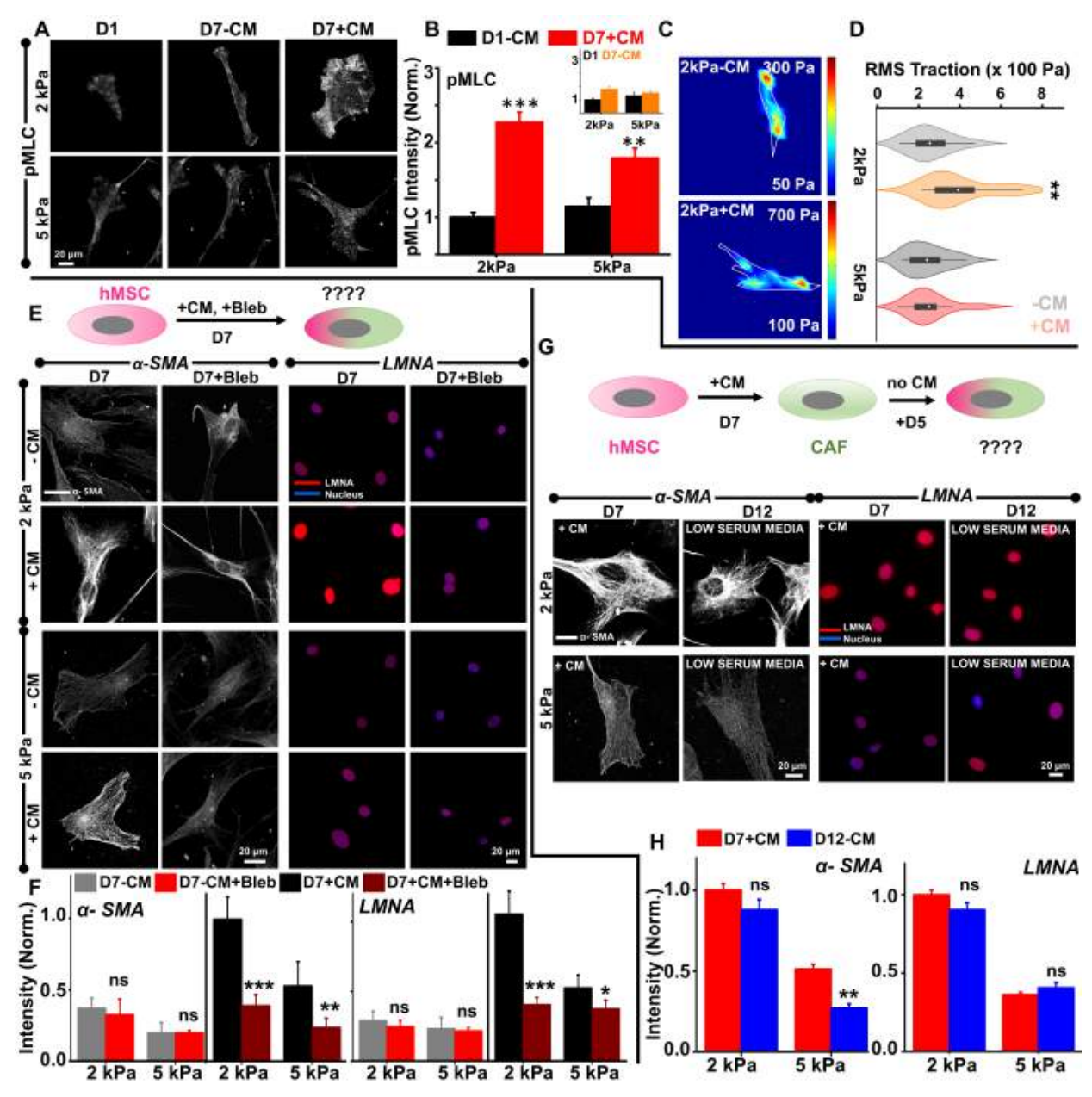

Figure 4: $2 \mathrm{kPa}$ CAFs are more contractile and exhibit stable differentiation: A, B) Representative images and intensity quantification of pMLC staining in MSCs cultured $\pm \mathrm{CM}$ on (2 and 5) kPa gels for 7 days. Scale bar $=20 \mu \mathrm{m}$. For quantification of pMLC intensity, integrated intensities were normalized with respect to 2 $\mathrm{kPa},-\mathrm{CM}$, D1 condition ( $n \geq$ cells per condition pooled from $N=3$ independent experiments; error bars represent $\pm \mathrm{SEM}, * * *, * *$ indicates statistical significance $(p<0.001, p<0.01$, respectively) compared to $\mathrm{D} 1-\mathrm{CM})$. Insets show integrated intensities normalized to $2 \mathrm{kPa},-\mathrm{CM}$, D1 condition for cells cultured in the absence of $\mathrm{CM}$ on (2 and 5) kPa gels. C, D) Representative traction force heatmaps and RMS traction force quantification in MSCs cultured $\pm \mathrm{CM}$ on (2 and 5$) \mathrm{kPa}$ gels for 7 days $(n=12$ cells per condition from $N=3$ independent experiments; error bars represent $\pm \mathrm{SEM}, * *$ indicates statistical significance compared to D1CM). E) To probe the effect of myosin inhibition on MSC differentiation into CAFs, MSCs were cultured in CM supplemented with $5 \mu \mathrm{M}$ Blebbistatin (Bleb) for 7 days. Representative images and intensity quantification of $\alpha$-SMA/LMNA stained MSCs cultured on (2 and 5) kPa gels for 7 days \pm CM, \pm Bleb. Scale bar $=20 \mu \mathrm{m}$. F) Integrated intensities were normalized with respect to $2 \mathrm{kPa},+\mathrm{CM}$ condition $(n \geq 40$ cells per condition pooled from $N=3$ independent experiments; error bars represent $\pm \mathrm{SEM}, * * *, * *$ indicates statistical significance $(p<0.001, p<0.01$, respectively) compared to D7+CM condition). G, H) Experimental plan to probe the stability of CAF differentiation. After 7 days culture in the presence of CM, MSCs were cultured on (2 and 5 ) $\mathrm{kPa}$ gels for another 5 days in complete media and stained for CAF markers. Representative images and intensity quantification of $\alpha-S M A / L M N A$ staining after day 5 of CM removal (i.e., Day 12 (D12)) on $(2$ and 5$) \mathrm{kPa}$ gels (Scale bar: $20 \mu \mathrm{m}$ ). Integrated intensities were normalized with respect to $2 \mathrm{kPa},+\mathrm{CM}$, D7 condition $(n \geq 40$ cells per condition pooled from $N=3$ independent experiments; error bars represent $\pm \mathrm{SEM}, * *$ indicates statistical significance $(p<0.01)$ compared to D7+CM condition). 
bioRxiv preprint doi: https://doi.org/10.1101/2021.01.12.426460; this version posted January 13, 2021. The copyright holder for this preprint (which was not certified by peer review) is the author/funder, who has granted bioRxiv a license to display the preprint in perpetuity. It is made available under aCC-BY-NC-ND 4.0 International license.

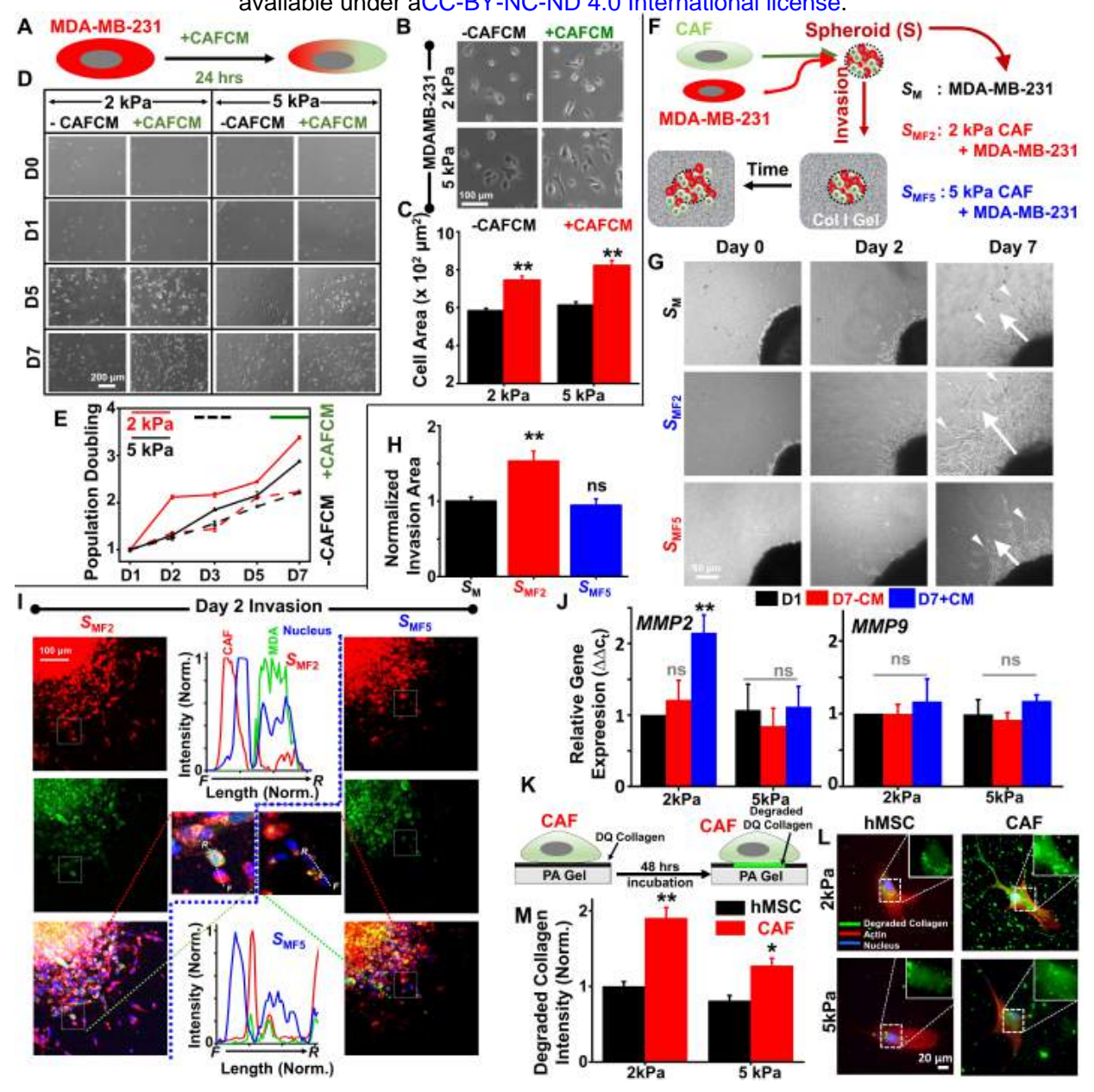

Figure 5: $2 \mathrm{kPa}$ CAFs maximally enhance proliferation and invasiveness of MDA-MB-231 breast cancer cells: A) Cancer cells were cultured in stiffness-modulated CAF secreted CM (CAFCM) for 24 hrs. B) Representative images of MDA-MB-231 cultured on (2 and 5) kPa gels \pm CAFCM. Scale bar $=100 \mu \mathrm{m}$. C) Quantification of MDA-MB-231 cell spread area \pm CAFCM $(n \geq 50$ cells per condition pooled from $N=3$ independent experiments; error bars represent $\pm \mathrm{SEM}, * *$ indicates statistical significance $(p<0.01)$ compared to -CM). D) Representative images of MDA-MB-231 cultured on (2 and 5) kPa gels \pm CAFCM for 7 days. Scale bar $=200 \mu \mathrm{m}$. E) Quantification of population doubling of cancer cells \pm CAFCM $(N=3$ independent experiments; error bars represent $\pm \mathrm{SEM})$. F) Schematic of spheroid formation using cancer cells alone $\left(S_{M}\right)$ and by combining cancer cells with (2 and 5) $\mathrm{kPa}$ CAFs $\left(\mathrm{S}_{M F 2}\right.$ and $\mathrm{S}_{M F 5}$, respectively). Spheroid invasion was performed by embedding spheroids in 3D collagen gels. G) Representative temporal images of invasion by $S_{M}, \mathrm{~S}_{M F 2}$ and $\mathrm{S}_{M F 5}$ spheroids in $3 \mathrm{D}$ collagen gels. Scale bar $=50 \mu \mathrm{m}$. White arrows depict the extent of outward invasion. H) Quantification of area invaded by $\mathrm{S}_{M F 2}$ and $\mathrm{S}_{M F 5}$ spheroids normalized to that of $S_{M}$ spheroids $(N=3$ independent experiments; error bars represent $\pm \mathrm{SEM} ; * *$ indicates statistical significance $(p<0.01)$ compared to $S_{M}$, ns indicates not significant). I) Spatial positioning of CAFs and MDA-MB-231 cells at the invasive front. CAFs were stained with CellTracker red and MDA-MB-231 with CellTracker green. Intensity profiles along lines drawn in the boxed regions show the relative positions of CAFs and MDA-MB231 cells from front (F) to rear (R). J) Expression profile of matrix degrading enzymes MMP2 and MMP9 in MSCs cultured on (2 and 5$) \mathrm{kPa}$ gels $\pm \mathrm{CM}$ for 7 days $(N=3$ independent experiments, $* *$ indicates statistical significance $(p<0.01)$ compared to D1, ns indicates not significant). K) Schematic of assessing MMP mediated matrix degradation. Cells were plated on gels functionalized with collagen mixed with DQ collagen. MMP mediated degradation was visualized by fluorescence signal. L, M) Representative images and intensity quantification of degraded collagen by MSCs on $2 \mathrm{kPa} / 5 \mathrm{kPa}$ gels and by $2 \mathrm{kPa} / 5 \mathrm{kPa} \mathrm{CAFs}$. Integrated intensities were normalized w.r.t $2 \mathrm{kPa}$ D1 MSCs $(n \geq 55$ cells per condition pooled from $N=3$ independent experiments; error bars represent $\pm \mathrm{SEM}, * *, *$ indicates statistical significance $(P<0.01,0.05)$ w.r.t D1 MSCs.) 
bioRxiv preprint doi: https://doi.org/10.1101/2021.01.12.426460; this version posted January 13, 2021. The copyright holder for this preprint (which was not certified by peer review) is the author/funder, who has granted bioRxiv a license to display the preprint in perpetuity. It is made available under aCC-BY-NC-ND 4.0 International license.

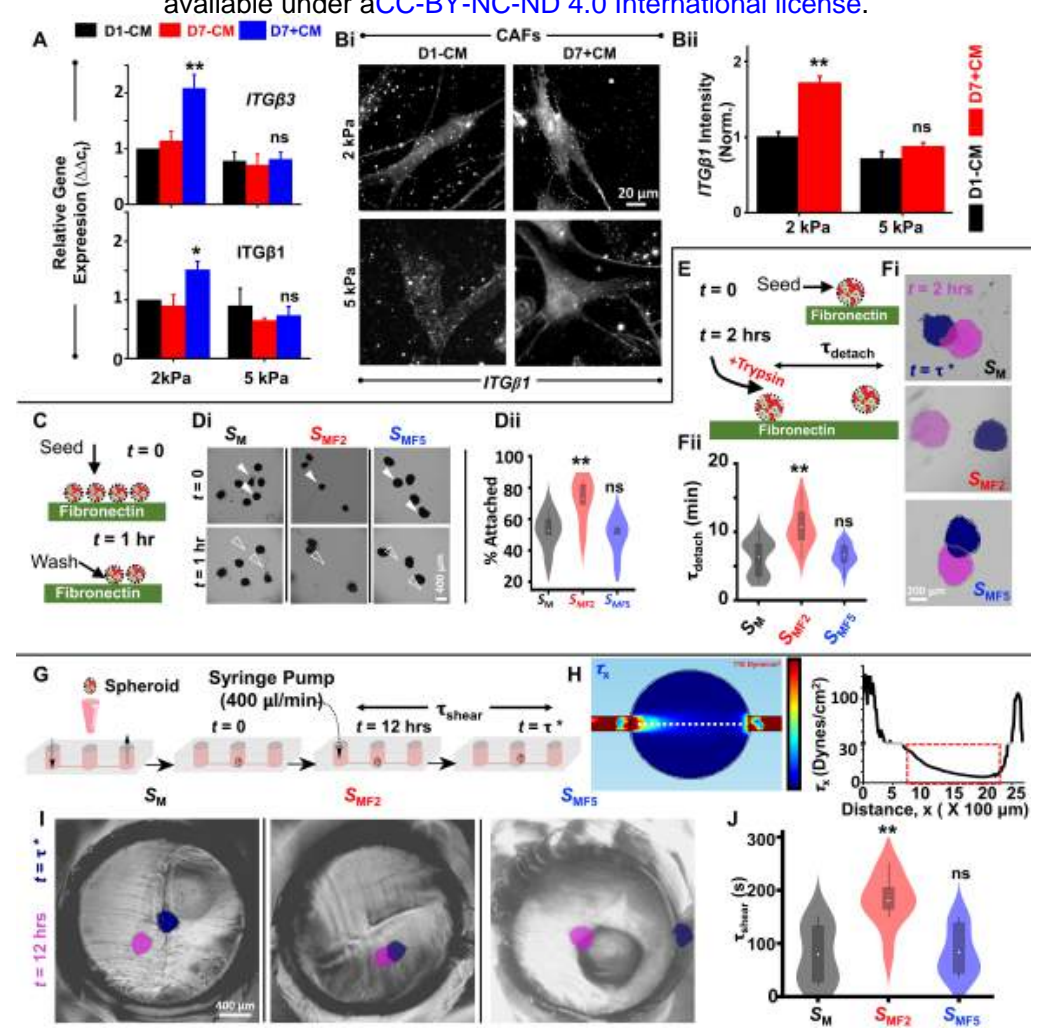

Figure 6: $2 \mathrm{kPa}$ heterospheroids are maximally adhesive and resistant to shear stresses: A) Expression profile of integrin $\beta 1$ (ITGB1) and integrin $\beta 3$ (ITGB3) in MSCs cultured on (2 and 5) $\mathrm{kPa}$ gels $\pm \mathrm{CM}$ for 7 days $(N=3$ independent experiments; error bars represent $\pm \mathrm{SEM}, * *, *$ indicate statistical significance $(p<$ $0.01, p<0.05)$ compared to D1-CM, ns indicates not significant). Bi, ii) Immunostaining and quantification of ITGB1 in MSCs (D1-CM) and CAFs (D7+CM) on 2 and $5 \mathrm{kPa}$ gels. Integrated intensities were normalized w.r.t $2 \mathrm{kPa}$ D1 MSCs $(n \geq 55$ cells per condition pooled from $N=3$ independent experiments; error bars represent $\pm \mathrm{SEM}, * *$ indicates statistical significance $(p<0.01)$ w.r.t D1-CM, ns indicates not significant.) C) Schematic of adhesion assay. $S_{M}, \mathrm{~S}_{M F 2}$ and $\mathrm{S}_{M F 5}$ spheroids were seeded on fibronectin (FN) coated coverslips and allowed to adhere for $t=1 \mathrm{hr}$. After removing loosely attached spheroids by washing with PBS, samples were imaged. Di) Representative images of spheroids at $t=0 \mathrm{hr}$ and $t=1 \mathrm{hr}$. Scale bar $=400$ $\mu \mathrm{m}$. Dii) Percentage of $S_{M}, \mathrm{~S}_{M F 2}$ and $\mathrm{S}_{M F 5}$ spheroids that remained attached $(n \geq 53$ spheroids per condition pooled from $N=3$ independent experiments; error bars represent $\pm \mathrm{SEM}$; $* *$ indicates statistical significance $(p<0.01)$ w.r.t $S_{M}$ spheroids, ns indicates not significant.). E) Schematic of de-adhesion assay. Spheroids seeded on FN coated coverslips were allowed to adhere for 2 hrs. After 2 hrs, trypsin was added to coverslips and spheroids were imaged till they were dislodged at time $t=\tau^{*}$ (i.e., moved from their initial position). $\tau_{\text {detach }}=t-120$ represents the duration (in minutes) required for a spheroid to de-adhere, and is a measure of adhesion strength. Fi, ii) Representative images of spheroids at $t=2 \mathrm{hrs}$ (pink) and at $t=\tau^{*}$ (blue) and quantification of $\tau_{\text {detach }}(n \geq 14$ spheroids per condition pooled from $N=3$ independent experiments; error bars represent $\pm \mathrm{SEM} ; * *$ indicates statistical significance $(p<0.01)$ w.r.t $S_{M}$ spheroids, ns indicates not significant). Scale bar $=200 \mu \mathrm{m}$. G) Experimental setup for assessing adhesion strength of individual spheroids under shear stresses. Spheroids were seeded in the middle port of a straight channel and allowed to attach for 10-12 hrs. Subsequently, spheroids were subjected to a flow rate of $400 \mu \mathrm{l} / \mathrm{min}$ using a mechanical syringe pump until the spheroids detached from the substrate. H) Spatial map of shear stresses generated in the middle port of the device simulated in COMSOL using laminar flow physics. Graph represents variation of shear stress along the white dotted line. I) Representative images of spheroids after adhesion at $t=12$ hrs (pink) and at the time of detachment $\left(t=\tau^{*}\right.$, blue). Scale bar $=200 \mu \mathrm{m}$. J) Quantification of time required for detachment of spheroids under shear $\left(\tau_{\text {shear }}\right)(n \geq 7$ spheroids per condition pooled from $N=3$ independent experiments; error bars represent $\pm \mathrm{SEM} ; * *$ indicates statistical significance $(p<0.01)$ w.r.t $S_{M}$, ns indicates not significant). 


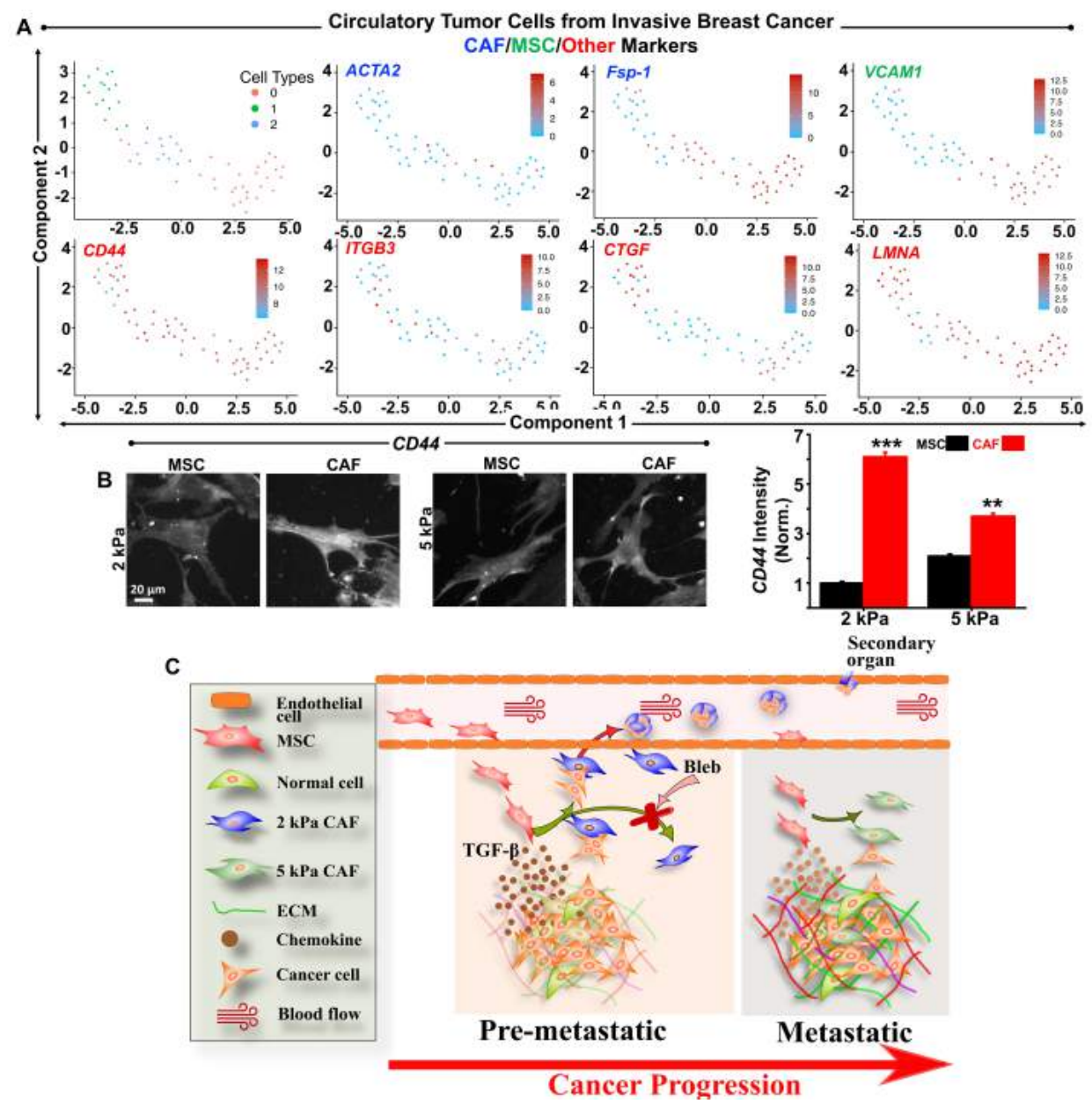

Figure 7: Presence of CAFs in circulating tumor cell (CTC) clusters and proposed model of pre-metastatic niche as a driver of cancer progression: A) scRNA sequencing from invasive breast cancer patients and MMTVPyMT mouse mmodels. 61 CTCs were clustered into 3 cell types. Each cell cluster expressed MSC and CAF markers. Other than these markers CTCs also expressed CD44 (Cohesion), ITGB3 (Adhesion), LMNA (Differentiation) and CTGF (Bone metastasis). B) Immunostaining and quantification of CD44 in MSCs (D1-CM) and CAFs (D7+CM) on 2 and $5 \mathrm{kPa}$ gels. Integrated intensities were normalized with respect to 2 $\mathrm{kPa}$ D1 MSCs $(n \geq 55$ cells per condition pooled from $N=3$ independent experiments; error bars represent $\pm \mathrm{SEM}, * * *, * *$ indicate statistical significance $(p<0.001, p<0.01$, respectively) w.r.t D1-CM). C) Factors secreted by cancer cells on pre-metastatic stroma mimetic $2 \mathrm{kPa}$ gels optimally induce MSC recruitment at the site of tumors and differentiation into CAFs. In addition to driving cancer cell proliferation through secreted soluble factors, differentiated CAFs aid in stromal invasion as well as distant metastasis by increasing adhesivity of cancer cells. 